\title{
Some Class of Third- and Fourth-Order Iterative Methods for Solving Nonlinear Equations
}

\author{
J. P. Jaiswal \\ Department of Mathematics, Maulana Azad National Institute of Technology, Bhopal 462051, India \\ Correspondence should be addressed to J. P. Jaiswal; asstprofjpmanit@gmail.com
}

Received 30 July 2013; Revised 11 January 2014; Accepted 25 February 2014; Published 11 May 2014

Academic Editor: Malgorzata Peszynska

Copyright ( 2014 J. P. Jaiswal. This is an open access article distributed under the Creative Commons Attribution License, which permits unrestricted use, distribution, and reproduction in any medium, provided the original work is properly cited.

\begin{abstract}
The object of the present work is to give the new class of third- and fourth-order iterative methods for solving nonlinear equations. Our proposed third-order method includes methods of Weerakoon and Fernando (2000), Homeier (2005), and Chun and Kim (2010) as particular cases. The multivariate extension of some of these methods has been also deliberated. Finally, some numerical examples are given to illustrate the performances of our proposed methods by comparing them with some well existing third- and fourth-order methods. The efficiency of our proposed fourth-order method over some fourth-order methods is also confirmed by basins of attraction.
\end{abstract}

\section{Introduction}

Solving nonlinear equations is one of the most important problems in numerical analysis. To solve nonlinear equations, some iterative methods (such as Secant method and Newton method) are usually used. Throughout this paper, we consider iterative methods to find a simple root $\alpha$ of a nonlinear equation $f(x)=0$. It is well known that the order of convergence of the Newton method is two. To improve the efficiency of the Newton method, many modified thirdorder methods have been presented in the literature by using different techniques. Weerakoon and Fernando in [1] obtained a third-order method by approximating the integral in Newton's theorem by trapezoidal rule, Homeier in [2] by using inverse function theorem, Chun and Kim in [3] by using circle of curvature concept, and so forth. Kung and Traub [4] presented a hypothesis on the optimality of the iterative methods by giving $2^{n-1}$ as the optimal order. This means that the Newton method involved two function evaluations per iteration and is optimal with 1.414 as the efficiency index. By taking into account the optimality concept, many authors are trying to build iterative methods of optimal (higher) order of convergence.

The convergence order of the above discussed methods is three with three (one function and two derivatives) evaluations per full iteration. Clearly, its efficiency index $\left(3^{1 / 3} \approx 1.442\right)$ is not high (optimal). In recent days, authors are improving these types of nonoptimal order methods to optimal order by using different techniques, such as in [5] by using linear combination of two third-order methods and in [6] by using polynomial approximations. Recently, Soleymani et al. [7] have used two different weight functions in the methods of $[1,2]$ to make them optimal.

This paper is organized as follows: in Section 2, we describe a new class of third-order iterative methods by using the concept of weight function which includes the methods of [1-3]. After that, order of this class of methods has been accelerated from three to four by introducing one more weight function and without adding more function evaluations. Section 3 is devoted to the extension of some proposed methods to the multivariate case. Finally, we give some numerical examples and the new methods are compared with some existing third- and fourth-order methods. Efficiency of our proposed fourth-order method is shown by basins of attraction.

\section{Methods and Convergence Analysis}

Before constructing the methods, here we state the following definitions. 
Definition 1. Let $f(x)$ be a real valued function with a simple root $\alpha$ and let $x_{n}$ be a sequence of real numbers that converge towards $\alpha$. The order of convergence $m$ is given by

$$
\lim _{n \rightarrow \infty} \frac{x_{n+1}-\alpha}{\left(x_{n}-\alpha\right)^{m}}=\zeta \neq 0,
$$

where $\zeta$ is the asymptotic error constant and $m \in R^{+}$.

Definition 2. Let $n$ be the number of function evaluations of the new method. The efficiency of the new method is measured by the concept of efficiency index $[8,9]$ and defined as

$$
m^{1 / n}
$$

where $m$ is the order of convergence of the new method.

2.1. Third-Order Methods. In this section, we construct a new class of two-step third-order iterative methods. Let us consider the following iterative formula:

$$
\begin{gathered}
y_{n}=x_{n}-\frac{f\left(x_{n}\right)}{f^{\prime}\left(x_{n}\right)}, \\
x_{n+1}=x_{n}-A(t) \frac{f\left(x_{n}\right)}{f^{\prime}\left(x_{n}\right)},
\end{gathered}
$$

where $t=f^{\prime}\left(y_{n}\right) / f^{\prime}\left(x_{n}\right)$. The following theorem indicates under what conditions on the weight function in (3) the order of convergence is three.

Theorem 3. Let the function $f$ have sufficient number of continuous derivatives in a neighborhood of $\alpha$ which is a simple root of $f$; then method (3) has third-order convergence, when the weight function $A(t)$ satisfies the following conditions:

$$
A(1)=1, \quad A^{\prime}(1)=-\frac{1}{2}, \quad\left|A^{\prime \prime}(1)\right|<+\infty .
$$

Proof. Let $e_{n}=x_{n}-\alpha$ be the error in the $n$th iterate and $c_{h}=f^{(h)}(\alpha) / h !, h=1,2,3, \ldots$. We provide Taylor's series expansion of each term involved in (3). By Taylor series expansion around the simple root in the $n$th iteration, we have

$$
\begin{gathered}
f\left(x_{n}\right)=f^{\prime}(\alpha)\left[e_{n}+c_{2} e_{n}^{2}+c_{3} e_{n}^{3}+c_{4} e_{n}^{4}+c_{5} e_{n}^{5}\right]+O\left(e_{n}^{6}\right), \\
f^{\prime}\left(x_{n}\right)=f^{\prime}(\alpha)\left[1+2 c_{2} e_{n}+3 c_{3} e_{n}^{2}+4 c_{4} e_{n}^{3}+5 c_{5} e_{n}^{4}\right]+O\left(e_{n}^{5}\right) .
\end{gathered}
$$

Furthermore, it can be easily found that

$$
\frac{f\left(x_{n}\right)}{f^{\prime}\left(x_{n}\right)}=e_{n}-c_{2} e_{n}^{2}+\left(2 c_{2}^{2}-2 c_{3}\right) e_{n}^{3}+O\left(e_{n}^{4}\right) \text {. }
$$

By considering this relation, we obtain

$$
y_{n}=\alpha+c_{2} e_{n}^{2}+2\left(c_{3}-c_{2}^{2}\right) e_{n}^{3}+O\left(e_{n}^{4}\right)
$$

At this time, we should expand $f^{\prime}\left(y_{n}\right)$ around the root by taking into consideration (7). Accordingly, we have

$$
f^{\prime}\left(y_{n}\right)=f^{\prime}(\alpha)\left[1+2 c_{2}^{2} e_{n}^{2}+\left(4 c_{2} c_{3}-4 c_{2}^{3}\right) e_{n}^{3}+O\left(e_{n}^{4}\right)\right] .
$$

Furthermore, we have

$$
\frac{f^{\prime}\left(y_{n}\right)}{f^{\prime}\left(x_{n}\right)}=1-2 c_{2} e_{n}+\left(6 c_{2}^{2}-3 c_{3}\right) e_{n}^{2}+\cdots+O\left(e_{n}^{4}\right) \text {. }
$$

By virtue of (9) and (4), we attain

$$
\begin{aligned}
A(t) \times \frac{f\left(x_{n}\right)}{f^{\prime}\left(x_{n}\right)}= & e_{n}-\frac{1}{2} \\
& \times\left[c_{3}-4 c_{2}^{2}\left(-1+A^{\prime \prime}(1)\right)\right] e_{n}^{3}+O\left(e_{n}^{4}\right) .
\end{aligned}
$$

Finally, using (10) in the second step of (3), we have the following error equation:

$$
\begin{aligned}
e_{n+1} & =x_{n+1}-\alpha \\
& =x_{n}-A(t) \times \frac{f\left(x_{n}\right)}{f^{\prime}\left(x_{n}\right)}-\alpha \\
& =\frac{1}{2}\left[c_{3}-4 c_{2}^{2}\left(-1+A^{\prime \prime}(1)\right)\right] e_{n}^{3}+O\left(e_{n}^{4}\right),
\end{aligned}
$$

which has the third order of convergence. This proves the theorem.

\section{Particular Cases}

Case 1. If we take $A(t)=2 /(1+t)$ in (3), then we get the formula

$$
\begin{gathered}
y_{n}=x_{n}-\frac{f\left(x_{n}\right)}{f^{\prime}\left(x_{n}\right)}, \\
x_{n+1}=x_{n}-\frac{2 f\left(x_{n}\right)}{f^{\prime}\left(x_{n}\right)+f^{\prime}\left(y_{n}\right)},
\end{gathered}
$$

which is the same as established by Weerakoon and Fernando in [1].

Case 2. If we take $A(t)=(t+1) / 2 t$ in (3), then we get the formula

$$
\begin{gathered}
y_{n}=x_{n}-\frac{f\left(x_{n}\right)}{f^{\prime}\left(x_{n}\right)}, \\
x_{n+1}=x_{n}-\frac{f\left(x_{n}\right)}{2}\left(\frac{1}{f^{\prime}\left(x_{n}\right)}+\frac{1}{f^{\prime}\left(y_{n}\right)}\right),
\end{gathered}
$$

which is the same as established by Homeier in [2]. 
Case 3. If we take $A(t)=(3-t) / 2$ in (3), then we get the formula

$$
\begin{gathered}
y_{n}=x_{n}-\frac{f\left(x_{n}\right)}{f^{\prime}\left(x_{n}\right)}, \\
x_{n+1}=x_{n}-\frac{1}{2}\left(3-\frac{f^{\prime}\left(y_{n}\right)}{f^{\prime}\left(x_{n}\right)}\right) \frac{f\left(x_{n}\right)}{f^{\prime}\left(x_{n}\right)},
\end{gathered}
$$

which is the same as established by Chun and Kim in [3].

Case 4. If we take $A(t)=2 t /(3 t-1)$ in (3), then we get the formula

$$
\begin{gathered}
y_{n}=x_{n}-\frac{f\left(x_{n}\right)}{f^{\prime}\left(x_{n}\right)}, \\
x_{n+1}=x_{n}-\left(\frac{2 f^{\prime}\left(y_{n}\right)}{3 f^{\prime}\left(y_{n}\right)-f^{\prime}\left(x_{n}\right)}\right) \frac{f\left(x_{n}\right)}{f^{\prime}\left(x_{n}\right)},
\end{gathered}
$$

and its error expression is given by

$$
e_{n+1}=\frac{1}{2}\left[-2 c_{2}^{2}+c_{3}\right] e_{n}^{3}+O\left(e_{n}^{4}\right) \text {. }
$$

Remark 4. By taking suitable weight function $A(t)$ in (3) which satisfies the conditions of (4), one can get number of third-order methods.

2.2. Fourth-Order Methods. The convergence order of the previous class of methods is three with three (one function and two derivatives) evaluations per full iteration. Clearly its efficiency index $\left(3^{1 / 3} \approx 1.442\right)$ is not high (optimal) so we use one more weight function in the second step of (3) and introduce a constant in its first step. Thus, we consider

$$
\begin{gathered}
y_{n}=x_{n}-a \cdot \frac{f\left(x_{n}\right)}{f^{\prime}\left(x_{n}\right)}, \\
x_{n+1}=x_{n}-\{P(t) \times Q(t)\} \frac{f\left(x_{n}\right)}{f^{\prime}\left(x_{n}\right)},
\end{gathered}
$$

where $P(t)$ and $Q(t)$ are real-valued weight functions with $t=$ $f^{\prime}\left(y_{n}\right) / f^{\prime}\left(x_{n}\right)$ and $a$ is a real constant. The following theorem indicates under what conditions on the weight functions and constant $a$ in (17) the order of convergence will arrive at the optimal level four.

Theorem 5. Let the function $f$ have sufficient number of continuous derivatives in a neighborhood of $\alpha$ which is a simple root of $f$; then method (17) has fourth-order convergence, when $a=2 / 3$ and the weight functions $P(t)$ and $Q(t)$ satisfy the following conditions:

$$
\begin{gathered}
P(1)=1, \quad P^{\prime}(1)=-\frac{1}{2}, \quad\left|P^{(3)}(1)\right|<\infty, \\
Q(1)=1, \quad Q^{\prime}(1)=-\frac{1}{4}, \quad Q^{\prime \prime}(1)=2-P^{\prime \prime}(1), \\
\left|Q^{(3)}(1)\right|<\infty .
\end{gathered}
$$

Proof. Using (5) and $a=2 / 3$ in the first step of (17), we have

$$
y_{n}=\alpha+\frac{e_{n}}{3}+\frac{2 c_{2} e_{n}^{2}}{3}+\frac{4\left(c_{3}-c_{2}^{2}\right) e_{n}^{3}}{3}+\cdots+O\left(e_{n}^{5}\right) .
$$

Now we should expand $f^{\prime}\left(y_{n}\right)$ around the root by taking into consideration (19). Thus, we have

$$
f^{\prime}\left(y_{n}\right)=f^{\prime}(\alpha)\left[1+\frac{2 c_{2} e_{n}}{3}+\frac{\left(4 c_{2}^{2}+c_{3}\right) e_{n}^{2}}{3}+\cdots+O\left(e_{n}^{5}\right)\right]
$$

Furthermore, we have

$$
\frac{f^{\prime}\left(y_{n}\right)}{f^{\prime}\left(x_{n}\right)}=1-\frac{2 c_{2}}{3} e_{n}+\left(4 c_{2}^{2}-\frac{8 c_{3}}{3}\right) e_{n}^{2}+\cdots+O\left(e_{n}^{5}\right)
$$

By virtue of (21) and (18), we can obtain

$$
\begin{aligned}
\{P(t) \times Q(t)\} \frac{f\left(x_{n}\right)}{f^{\prime}\left(x_{n}\right)} & \\
= & e_{n}-\frac{1}{81} \\
\times & {\left[-81 c_{2} c_{3}+9 c_{4}\right.} \\
& +\left(309+24 A^{\prime \prime}(1)+32 A^{(3)}(1)\right. \\
& \left.\left.\quad+32 B^{(3)}(1)\right) c_{2}^{3}\right] e_{n}^{4}+O\left(e_{n}^{5}\right) .
\end{aligned}
$$

Finally, using (22) in the second step of (17), we have the following error expression:

$$
\begin{aligned}
e_{n+1}= & x_{n+1}-\alpha \\
= & x_{n}-\{P(t) \times Q(t)\} \frac{f\left(x_{n}\right)}{f^{\prime}\left(x_{n}\right)}-\alpha \\
= & \frac{1}{81}\left[-81 c_{2} c_{3}+9 c_{4}\right. \\
& +\left(309+24 A^{\prime \prime}(1)+32 A^{(3)}(1)\right. \\
& \left.\left.+32 B^{(3)}(1)\right) c_{2}^{3}\right] e_{n}^{4}+O\left(e_{n}^{5}\right),
\end{aligned}
$$

which shows the fourth-order convergence. It confirms the result.

It is clear that our class of fourth-order iterative methods (17) requires three function evaluations per iteration, that is, one function and two first derivative evaluations. Thus our new class is optimal. Clearly its efficiency index is $4^{1 / 3}=$ 1.5874 (high). Now by choosing appropriate weight functions in (17) which satisfy the conditions of (18), one can give a number of optimal two-step fourth-order iterative methods. 


\section{Particular Cases}

Method 1. If we take $P(t)=2 /(1+t), Q(t)=2-(7 / 4) t+$ $(3 / 4) t^{2}$, and $a=2 / 3$ in (17), then we get the following method:

$$
\begin{gathered}
y_{n}=x_{n}-\frac{2}{3} \frac{f\left(x_{n}\right)}{f^{\prime}\left(x_{n}\right)}, \\
x_{n+1}=x_{n}-\left[2-\frac{7}{4} \frac{f^{\prime}\left(y_{n}\right)}{f^{\prime}\left(x_{n}\right)}+\frac{3}{4}\left(\frac{f^{\prime}\left(y_{n}\right)}{f^{\prime}\left(x_{n}\right)}\right)^{2}\right] \\
\times \frac{2 f\left(x_{n}\right)}{f^{\prime}\left(x_{n}\right)+f^{\prime}\left(y_{n}\right)},
\end{gathered}
$$

and its error equation is given by

$$
e_{n+1}=\frac{1}{9}\left[-9 c_{2} c_{3}+c_{4}+33 c_{2}^{3}\right] e_{n}^{4}+O\left(e_{n}^{5}\right) .
$$

Method 2. If we take $P(t)=(t+1) / 2 t, Q(t)=(7 / 4)-(5 / 4) t+$ $(1 / 2) t^{2}$, and $a=2 / 3$ in (17), then we get the following method:

$$
\begin{gathered}
y_{n}=x_{n}-\frac{2}{3} \frac{f\left(x_{n}\right)}{f^{\prime}\left(x_{n}\right)}, \\
x_{n+1}=x_{n}-\left[\frac{7}{4}-\frac{5}{4} \frac{f^{\prime}\left(y_{n}\right)}{f^{\prime}\left(x_{n}\right)}+\frac{1}{2}\left(\frac{f^{\prime}\left(y_{n}\right)}{f^{\prime}\left(x_{n}\right)}\right)^{2}\right] \\
\times \frac{f\left(x_{n}\right)}{2}\left(\frac{1}{f^{\prime}\left(x_{n}\right)}+\frac{1}{f^{\prime}\left(y_{n}\right)}\right),
\end{gathered}
$$

and its error equation is given by

$$
e_{n+1}=\frac{1}{9}\left[-c_{2} c_{3}+\frac{c_{4}}{9}+\frac{79}{27} c_{2}^{3}\right] e_{n}^{4}+O\left(e_{n}^{5}\right) .
$$

Method 3. If we take $P(t)=(3-t) / 2, Q(t)=(9 / 4)-(9 / 4) t+t^{2}$, and $a=2 / 3$ in (17), then we get the following method:

$$
\begin{gathered}
y_{n}=x_{n}-\frac{2}{3} \frac{f\left(x_{n}\right)}{f^{\prime}\left(x_{n}\right)}, \\
x_{n+1}=x_{n}-\left[\frac{9}{4}-\frac{9}{4} \frac{f^{\prime}\left(y_{n}\right)}{f^{\prime}\left(x_{n}\right)}+\left(\frac{f^{\prime}\left(y_{n}\right)}{f^{\prime}\left(x_{n}\right)}\right)^{2}\right] \\
\times\left(\frac{3}{2}-\frac{f^{\prime}\left(y_{n}\right)}{2 f^{\prime}\left(x_{n}\right)}\right) \frac{f\left(x_{n}\right)}{f^{\prime}\left(x_{n}\right)},
\end{gathered}
$$

which is the same as given in [10].

Method 4. If we take $P(t)=2 t /(3 t-1), Q(t)=(3 / 2)-(3 / 4) t+$ $(1 / 4) t^{2}$, and $a=2 / 3$ in (17), then we get the following method:

$$
\begin{aligned}
y_{n}=x_{n} & -\frac{2}{3} \frac{f\left(x_{n}\right)}{f^{\prime}\left(x_{n}\right)}, \\
x_{n+1}=x_{n} & -\left[\frac{3}{2}-\frac{3}{4} \frac{f^{\prime}\left(y_{n}\right)}{f^{\prime}\left(x_{n}\right)}+\frac{1}{4}\left(\frac{f^{\prime}\left(y_{n}\right)}{f^{\prime}\left(x_{n}\right)}\right)^{2}\right] \\
& \times\left(\frac{2 f^{\prime}\left(y_{n}\right)}{3 f^{\prime}\left(y_{n}\right)-f^{\prime}\left(x_{n}\right)}\right) \frac{f\left(x_{n}\right)}{f^{\prime}\left(x_{n}\right)},
\end{aligned}
$$

and its error equation is given by

$$
e_{n+1}=\left[\frac{43}{27} c_{2}^{3}-c_{2} c_{3}+\frac{c_{4}}{9}\right] e_{n}^{4}+O\left(e_{n}^{5}\right)
$$

Remark 6. By choosing the appropriate weight functions in (17) which satisfy the conditions of (18), one can get number of fourth-order methods.

\section{Extension to Multivariate Case}

In this section, we extend some methods of the previous sections (similarly other methods can also be extended) to the multivariate case. Method (15) for systems of nonlinear equations can be written as

$$
\begin{aligned}
& Y^{(k)}=X^{(k)}-\left[F^{\prime}\left(X^{(k)}\right)\right]^{-1} F\left(X^{(k)}\right) \\
X^{(k+1)}= & X^{(k)}-2\left[3 F^{\prime}\left(Y^{(k)}\right)-F^{\prime}\left(X^{(k)}\right)\right]^{-1}\left[F^{\prime}\left(Y^{(k)}\right)\right] \\
& \cdot\left[F^{\prime}\left(X^{(k)}\right)\right]^{-1} F\left(X^{(k)}\right)
\end{aligned}
$$

where $X^{(k)}=\left[x_{1}^{(k)}, x_{2}^{(k)}, \ldots, x_{n}^{(k)}\right]^{T},(k=0,1,2, \ldots)$; similarly $Y^{(k)} ; I$ is $n \times n$ identity matrix; $F\left(X^{(k)}\right)=\left[f_{1}\left(x_{1}^{(k)}\right.\right.$, $\left.\left.x_{2}^{(k)}, \ldots, x_{n}^{(k)}\right), f_{2}\left(x_{1}^{(k)}, x_{2}^{(k)}, \ldots, x_{n}^{(k)}\right), \ldots, f_{n}\left(x_{1}^{(k)}, x_{2}^{(k)}, \ldots, x_{n}^{(k)}\right)\right]$; and $F^{\prime}\left(X^{(k)}\right)$ is the Jacobian matrix of $F$ at $X^{(k)}$.

Let $\alpha+H \in \Re^{n}$ be any point of the neighborhood of exact root $\alpha \in \mathfrak{R}^{n}$ of the nonlinear system $F(X)=0$. If Jacobian matrix $F^{\prime}(\alpha)$ is nonsingular, then Taylor's series expansion for multivariate case is

$$
\begin{aligned}
F(\alpha+H)= & F^{\prime}(\alpha)\left[H+C_{2} H^{2}+C_{3} H^{3}+\cdots+C_{p-1} H^{p-1}\right] \\
& +O\left(H^{p}\right),
\end{aligned}
$$

where $C_{i}=\left[F^{\prime}(\alpha)\right]^{-1}\left(F^{(i)}(\alpha) / i !\right), i \geq 2$, and

$$
\begin{aligned}
F^{\prime}(\alpha+H)=F^{\prime}(\alpha)[ & I+2 C_{2} H+3 C_{3} H^{2} \\
& \left.+\cdots+(p-1) C_{p-1} H^{p-2}\right]+O\left(H^{p-1}\right),
\end{aligned}
$$

where $I$ is an identity matrix. From the above equation, we can find

$$
\begin{aligned}
{\left[F^{\prime}(\alpha+H)\right]^{-1}=\left[F^{\prime}(\alpha)\right]^{-1}[} & I+A_{1} H+A_{2} H^{2} \\
& \left.+\cdots+A_{p-2} H^{p-2}\right]+O\left(H^{p-1}\right),
\end{aligned}
$$

where $A_{1}=-2 C_{2}, A_{2}=4 C_{2}^{2}-3 C_{3}, A_{3}=-8 C_{2}^{3}+6 C_{2} C_{3}+$ $6 C_{3} c_{2}-4 C_{4}$, and so on. Here we denote the error at $k$ th iterate by $E^{(k)}$; that is, $E^{(k)}=X^{(k)}-\alpha$. Now the order of convergence of method (31) is confirmed by the following theorem. 
Theorem 7. Let $F: D \subseteq \mathfrak{R}^{n} \rightarrow \mathfrak{R}^{n}$ be sufficiently Frechet differentiable in a convex set $D$ containing a root $\alpha$ of $F(X)=0$. Let us suppose that $F^{\prime}(X)$ is continuous and nonsingular in $D$ and $X^{(0)}$ is close to $\alpha$. Then the sequence $\left\{X^{(k)}\right\}_{k \geq 0}$ obtained by the iterative expression (31) converges to $\alpha$ with order three.

Proof. From (32), (33), and (34), we have

$$
\begin{aligned}
F\left(X^{(k)}\right)= & F^{\prime}(\alpha)\left[E^{(k)}+C_{2} E^{(k)^{2}}+C_{3} E^{(k)^{3}}+C_{4} E^{(k)^{4}}\right] \\
& +O\left(E^{(k)^{5}}\right)
\end{aligned}
$$

$$
\begin{aligned}
F^{\prime}\left(X^{(k)}\right)=F^{\prime}(\alpha)[ & I+2 C_{2} E^{(k)}+3 C_{3} E^{(k)^{2}} \\
& \left.+4 C_{4} E^{(k)^{3}}+5 C_{5} E^{(k)^{4}}\right]+O\left(E^{(k)^{5}}\right),
\end{aligned}
$$

$$
\begin{aligned}
{\left[F^{\prime}\left(X^{(k)}\right)\right]^{-1}=} & {\left[F^{\prime}(\alpha)\right]^{-1} } \\
& \times\left\{I-2 C_{2} E^{(k)}+\left(4 C_{2}^{2}-3 C_{3}\right) E^{(k)^{2}}\right. \\
& \left.+\left(-8 C_{2}^{3}+6 C_{2} C_{3}+6 C_{3} C_{2}-4 C_{4}\right) E^{(k)^{3}}\right\} \\
+ & O\left(E^{(k)^{4}}\right),
\end{aligned}
$$

where $C_{i}=\left[F^{\prime}(\alpha)\right]^{-1}\left(F^{(i)}(\alpha) / i !\right), i=2,3, \ldots$ Now from (37) and (35), we can obtain

$$
\begin{aligned}
& {\left[F^{\prime}\left(X^{(k)}\right)\right]^{-1} F\left(X^{(k)}\right)} \\
& \quad=E^{(k)}-C_{2} E^{(k)^{2}}+\left(2 C_{2}^{2}-2 C_{3}\right) E^{(k)^{3}}+O\left(E^{(k)^{4}}\right) .
\end{aligned}
$$

By virtue of (38), the first step of method (31) becomes

$$
Y^{(k)}=\alpha+C_{2} E^{(k)^{2}}+\left(-2 C_{2}^{2}+2 C_{3}\right) E^{(k)^{3}}+O\left(E^{(k)^{4}}\right) .
$$

Now the Taylor expansion for Jacobian matrix $F^{\prime}\left(Y^{(k)}\right)$ is given by

$$
\begin{aligned}
F^{\prime}\left(Y^{(k)}\right)=F^{\prime}(\alpha)[ & I+2 C_{2}^{2} E^{(k)^{2}} \\
& \left.-\left(4 C_{2}^{4}-4 C_{2} C_{3}\right) E^{(k)^{3}}\right]+O\left(E^{(k)^{4}}\right) .
\end{aligned}
$$

Therefore

$$
\begin{aligned}
& {\left[F^{\prime}\left(X^{(k)}\right)\right]^{-1} F^{\prime}\left(Y^{(k)}\right)} \\
& =I-2 C_{2} E^{(k)}+\left(6 C_{2}^{2}-3 C_{3}\right) E^{(k)^{2}} \\
& \quad+\left(-4 C_{2}^{4}-12 C_{2}^{3}+10 C_{2} C_{3}+6 C_{3} C_{2}-4 C_{4}\right) E^{(k)^{3}} \\
& \quad+O\left(E^{(k)^{4}}\right) \\
& {\left[3 F^{\prime}\left(Y^{(k)}\right)-F^{\prime}\left(X^{(k)}\right)\right]} \\
& =F^{\prime}(\alpha)\left[2 I-2 C_{2} E^{(k)}+\left(6 C_{2}^{2}-3 C_{3}\right) E^{(k)^{2}}\right. \\
& \quad+\left(-12 C_{2}^{4}-36 C_{2}^{3}+30 C_{2} C_{3}+18 C_{3} C_{2}-16 C_{4}\right) \\
& \left.\quad \times E^{(k)^{3}}\right]+O\left(E^{(k)^{4}}\right) .
\end{aligned}
$$

The above equation implies

$$
\begin{aligned}
& {\left[3 F^{\prime}\left(Y^{(k)}\right)-F^{\prime}\left(X^{(k)}\right)\right]^{-1}} \\
& =\frac{\left[F^{\prime}(\alpha)\right]^{-1}}{2}\left[I+B_{1} E^{(k)}+B_{2} E^{(k)^{2}}+B_{3} E^{(k)^{3}}\right] \\
& \quad+O\left(E^{(k)^{4}}\right),
\end{aligned}
$$

where

$$
\begin{gathered}
B_{1}=C_{2}, \\
B_{2}=-2 C_{2}^{2}+\frac{3}{2} C_{3}, \\
B_{3}=6 C_{2}^{4}+13 C_{2}^{3}-\frac{27}{2} C_{2} C_{3}-\frac{15}{2} C_{3} C_{2}+8 C_{4} .
\end{gathered}
$$

Now from (40) and (42), we have

$$
\begin{aligned}
& {\left[3 F^{\prime}\left(Y^{(k)}\right)-F^{\prime}\left(X^{(k)}\right)\right]^{-1} F^{\prime}\left(Y^{(k)}\right)} \\
& =\frac{1}{2}\left[I+E^{(k)}+\frac{3}{2} C_{3} E^{(k)^{2}}\right. \\
& +\left(2 C_{2}^{4}+15 C_{2}^{3}-\frac{19}{3} C_{2} C_{3}\right. \\
& \left.\left.\quad-\frac{15}{2} C_{3} C_{2}+8 C_{4}\right) E^{(k)^{3}}\right]+O\left(E^{(k)^{4}}\right) .
\end{aligned}
$$

By using (44) and (38), it can be found that

$$
\begin{aligned}
2[ & \left.3 F^{\prime}\left(Y^{(k)}\right)-F^{\prime}\left(X^{(k)}\right)\right]^{-1} \\
& \times\left[F^{\prime}\left(Y^{(k)}\right)\right]\left[F^{\prime}\left(X^{(k)}\right)\right]^{-1} F\left(X^{(k)}\right) \\
= & E^{(k)}+\left(C_{2}^{2}-\frac{1}{2} C_{3}\right) E^{(k)^{3}}+O\left(E^{(k)^{4}}\right) .
\end{aligned}
$$


TABLE 1: Functions and their roots.

\begin{tabular}{lc}
\hline$f(x)$ & \multicolumn{1}{c}{$\alpha$} \\
\hline$f_{1}(x)=e^{-x}-1+\frac{x}{5}$ & $4.9651142317442763036 \ldots$ \\
$f_{2}(x)=\frac{x^{3}+2.87 x^{2}-10.28}{4.62}-x$ & $2.0021187789538272889 \ldots$ \\
$f_{3}(x)=\frac{x+\operatorname{Cos} x \operatorname{Sin} x}{\pi}-\frac{1}{4}$ & $0.4158555967898679887 \ldots$ \\
$f_{4}(x)=x e^{-x}-0.1$ & $0.1118325591589629648 \ldots$ \\
\hline
\end{tabular}

Finally, using (45) in the second step of (31), we see that the error expression can be expressed as

$$
E^{(k+1)}=\left(-C_{2}^{2}+\frac{C_{3}}{3}\right) E^{(k)^{3}}+O\left(E^{(k)^{4}}\right)
$$

which shows the theorem.

Similarly, method (29) for systems of nonlinear equations is given by

$$
\begin{gathered}
Y^{(k)}=X^{(k)}-\frac{2}{3}\left[F^{\prime}\left(X^{(k)}\right)\right]^{-1} F\left(X^{(k)}\right), \\
X^{(k+1)}=X^{(k)}-\left[\frac{3}{2} I-\frac{3}{4}\left[F^{\prime}\left(X^{(k)}\right)\right]^{-1} F^{\prime}\left(Y^{(k)}\right)\right. \\
\left.+\frac{1}{4}\left(\left[F^{\prime}\left(X^{(k)}\right)\right]^{-1} F^{\prime}\left(Y^{(k)}\right)\right)^{2}\right] \\
\cdot 2\left[\left(3 F^{\prime}\left(Y^{(k)}\right)-F^{\prime}\left(X^{(k)}\right)\right)\right]^{-1} \\
\cdot\left[F^{\prime}\left(Y^{(k)}\right)\right]\left[F^{\prime}\left(X^{(k)}\right)\right]^{-1} F\left(X^{(k)}\right) .
\end{gathered}
$$

The order of the convergence of this method is confirmed by the following theorem.

Theorem 8. Let $F: D \subseteq \mathfrak{R}^{n} \rightarrow \mathfrak{R}^{n}$ be sufficiently Frechet differentiable in a convex set $D$ containing a root $\alpha$ of $F(X)=0$. Let us suppose that $F^{\prime}(\mathrm{X})$ is continuous and nonsingular in $D$ and $X^{(0)}$ is close to $\alpha$. Then the sequence $\left\{X^{(k)}\right\}_{k \geq 0}$ obtained by the iterative expression (47) converges to $\alpha$ with order four.

Proof. Multiplying (37) by (35), we get

$$
\begin{aligned}
s= & {\left[F^{\prime}\left(X^{(k)}\right)\right]^{-1} F\left(X^{(k)}\right) } \\
= & E^{(k)}-C_{2} E^{(k)^{2}}+\left(2 C_{2}^{2}-2 C_{3}\right) E^{(k)^{3}} \\
& +\left(-4 C_{2}^{3}+4 C_{2} C_{3}+3 C_{3} C_{2}-3 C_{4}\right) E^{(k)^{4}} \\
& +O\left(E^{(k)^{5}}\right) .
\end{aligned}
$$

Substituting value of (48) in the first step of (47), we find

$$
\begin{aligned}
Y^{(k)}= & \frac{1}{3} E^{(k)}+\frac{2}{3} C_{2} E^{(k)^{2}}+\frac{4}{3}\left(-C_{2}^{2}+C_{3}\right) E^{(k)^{3}} \\
& +\frac{2}{3}\left(4 C_{2}^{3}-4 C_{2} C_{3}-3 C_{3} C_{2}+3 C_{4}\right) E^{(k)^{4}} \\
& +O\left(E^{(k)^{5}}\right) .
\end{aligned}
$$

By using (49), the Taylor expansion of Jacobian matrix $F^{\prime}\left(Y^{(k)}\right)$ can be written as

$$
\begin{aligned}
& F^{\prime}\left(Y^{(k)}\right) \\
& =F^{\prime}(\alpha)\left[I+\frac{2}{3} C_{2} E^{(k)}+\left(\frac{4}{3} C_{2}^{2}+\frac{1}{3} C_{3}\right) E^{(k)^{2}}\right. \\
& +\left(\frac{-8}{3} C_{2}^{3}+\frac{8}{3} C_{2} C_{3}+\frac{4}{3} C_{3} C_{2}+\frac{4}{27} C_{4}\right) E^{(k)^{3}} \\
& +\left(\frac{5}{81} C_{5}+\frac{16}{3} C_{2}^{4}-\frac{16}{3} C_{2}^{3} C_{3}-4 C_{2} C_{3} C_{2}\right. \\
& \left.\left.+4 C_{2} C_{4}-\frac{4}{3} C_{3} C_{2}^{2}+\frac{8}{3} C_{3}^{2}+\frac{8}{9} C_{4} C_{2}\right) E^{(k)^{4}}\right] \\
& +O\left(E^{(k)^{5}}\right)
\end{aligned}
$$

From (37) and (50), it is obtained that

$$
\begin{aligned}
t= & {\left[F^{\prime}\left(X^{(k)}\right)\right]^{-1} F^{\prime}\left(Y^{(k)}\right) } \\
= & I-\frac{4}{3} C_{2} E^{(k)}+\left(4 C_{2}^{2}-\frac{8}{3} C_{3}\right) E^{(k)^{2}} \\
& +\left(\frac{-32}{3} C_{2}^{3}+8 C_{2} C_{3}+\frac{16}{3} C_{3} C_{2}-\frac{104}{27} C_{4}\right) E^{(k)^{3}} \\
& +O\left(E^{(k)^{4}}\right) .
\end{aligned}
$$

The above equation implies that

$$
\begin{aligned}
t^{2}= & \left(\left[F^{\prime}\left(X^{(k)}\right)\right]^{-1} F^{\prime}\left(Y^{(k)}\right)\right)^{2} \\
= & I-\frac{8}{3} C_{2} E^{(k)}+\left(\frac{88}{9} C_{2}^{2}-\frac{16}{3} C_{3}\right) E^{(k)^{2}} \\
& +\left(-32 C_{2}^{3}+\frac{176}{9} C_{2} C_{3}+\frac{128}{9} C_{3} C_{2}\right. \\
& \left.-\frac{208}{27} C_{4}\right) E^{(k)^{3}}+O\left(E^{(k)^{4}}\right) .
\end{aligned}
$$


TABLE 2: Comparison of the absolute error occurring in the estimates of the exact root.

\begin{tabular}{|c|c|c|c|c|c|}
\hline Function & Guess & Method & $\left|x_{1}-\alpha\right|$ & $\left|x_{2}-\alpha\right|$ & $\left|x_{3}-\alpha\right|$ \\
\hline \multirow{5}{*}{$f_{1}$} & \multirow{5}{*}{5} & NM & $0.21464 e-4$ & $0.83264 e-11$ & $0.12530 e-23$ \\
\hline & & WR3 & $0.11208 e-6$ & $0.37810 e-23$ & $0.14517 e-72$ \\
\hline & & HM3 & $0.12544 e-6$ & $0.59456 e-23$ & $0.63310 e-72$ \\
\hline & & $\mathrm{CH} 3$ & $0.98734 e-7$ & $0.22705 e-23$ & $0.27611 e-73$ \\
\hline & & M3 & $0.13882 e-6$ & $0.89316 e-23$ & $0.23790 e-71$ \\
\hline \multirow{5}{*}{$f_{2}$} & \multirow{5}{*}{2.5} & NM & $0.85925 e-1$ & $0.32675 e-2$ & $0.50032 e-5$ \\
\hline & & WR3 & $0.18271 e-1$ & $0.14770 e-5$ & $0.79610 e-18$ \\
\hline & & HM3 & $0.49772 e-2$ & $0.33027 e-8$ & $0.95318 e-27$ \\
\hline & & $\mathrm{CH} 3$ & $0.27815 e-1$ & $0.95903 e-5$ & $0.41254 e-15$ \\
\hline & & M3 & $0.14819 e-1$ & $0.64153 e-6$ & $0.51267 e-19$ \\
\hline \multirow{5}{*}{$f_{3}$} & \multirow{5}{*}{0.4} & NM & $0.10737 e-3$ & $0.50901 e-8$ & $0.11442 e-16$ \\
\hline & & WR3 & $0.20631 e-6$ & $0.53436 e-21$ & $0.92858 e-65$ \\
\hline & & HM3 & $0.52795 e-6$ & $0.19743 e-19$ & $0.10325 e-59$ \\
\hline & & $\mathrm{CH} 3$ & $0.93064 e-6$ & $0.20624 e-18$ & $0.22446 e-56$ \\
\hline & & M3 & $0.12723 e-5$ & $0.67803 e-18$ & $0.10261 e-54$ \\
\hline \multirow{5}{*}{$f_{4}$} & \multirow{5}{*}{0.3} & NM & $0.47567 e-1$ & $0.22849 e-2$ & $0.55356 e-5$ \\
\hline & & WR3 & $0.13039 e-1$ & $0.31800 e-5$ & $0.45048 e-16$ \\
\hline & & HM3 & $0.64393 e-3$ & $0.72236 e-10$ & $0.10226 e-30$ \\
\hline & & $\mathrm{CH} 3$ & $0.34012 e-1$ & $0.11125 e-3$ & $0.34855 e-11$ \\
\hline & & M3 & $0.75418 e-2$ & $0.36460 e-6$ & $0.41629 e-19$ \\
\hline
\end{tabular}

TABLE 3: Comparison of the absolute error occurring in the estimates of the exact root.

\begin{tabular}{|c|c|c|c|c|c|}
\hline Function & Guess & Method & $\left|x_{1}-\alpha\right|$ & $\left|x_{2}-\alpha\right|$ & $\left|x_{3}-\alpha\right|$ \\
\hline \multirow{4}{*}{$f_{1}$} & \multirow{4}{*}{5} & SL4 & $0.14780 e-5$ & $0.47702 e-23$ & $0.51761 e-93$ \\
\hline & & KH4 & $0.47426 e-9$ & $0.16796 e-40$ & $0.26418 e-166$ \\
\hline & & M4 I & $0.42743 e-9$ & $0.99425 e-41$ & $0.29108 e-167$ \\
\hline & & M4 II & $0.41059 e-9$ & $0.81178 e-41$ & $0.12404 e-167$ \\
\hline \multirow{4}{*}{$f_{2}$} & \multirow{4}{*}{2.5} & SL4 & $0.26594 e-1$ & $0.32982 e-6$ & $0.76311 e-26$ \\
\hline & & KH4 & $0.14965 e-1$ & $0.45484 e-7$ & $0.40826 e-29$ \\
\hline & & M4 I & $0.76770 e-2$ & $0.12105 e-8$ & $0.76261 e-36$ \\
\hline & & M4 II & $0.36004 e-2$ & $0.23379 e-10$ & $0.41876 e-43$ \\
\hline \multirow{4}{*}{$f_{3}$} & \multirow{4}{*}{0.4} & SL4 & $0.86290 e-7$ & $0.78612 e-28$ & $0.54150 e-112$ \\
\hline & & $\mathrm{KH} 4$ & $0.52074 e-7$ & $0.67319 e-29$ & $0.18803 e-116$ \\
\hline & & M4 I & $0.24363 e-7$ & $0.14724 e-30$ & $0.19642 e-123$ \\
\hline & & M4 II & $0.14232 e-7$ & $0.98181 e-32$ & $0.22236 e-128$ \\
\hline \multirow{4}{*}{$f_{4}$} & \multirow{4}{*}{0.3} & SL4 & $0.14507 e-1$ & $0.20259 e-6$ & $0.81662 e-26$ \\
\hline & & KH4 & $0.56586 e-1$ & $0.78841 e-4$ & $0.41658 e-15$ \\
\hline & & M4 I & $0.11886 e-1$ & $0.73037 e-7$ & $0.10950 e-27$ \\
\hline & & M4 II & $0.40617 e-2$ & $0.36373 e-9$ & $0.23749 e-37$ \\
\hline
\end{tabular}

Now from (51) and (52), we have

$$
\begin{aligned}
\frac{3}{2} I-\frac{3}{4} t+\frac{1}{4} t^{2}= & I+\frac{1}{3} C_{2} E^{(k)} \\
& +\left(\frac{-5}{9} C_{2}^{2}+\frac{2}{3} C_{3}\right) E^{(k)^{2}} \\
& +\left(-\frac{10}{9} C_{2} C_{3}-\frac{4}{9} C_{3} C_{2}+\frac{26}{27} C_{4}\right) E^{(k)^{3}} \\
& +O\left(E^{(k)^{4}}\right)
\end{aligned}
$$

Again by virtue of (50) and (36), it can be written as

$$
\begin{aligned}
& {\left[3 F^{\prime}\left(Y^{(k)}\right)-F^{\prime}\left(X^{(k)}\right)\right]} \\
& =F^{\prime}(\alpha)\left[2 I+\left(4 C_{2}^{2}-2 C_{3}\right) E^{(k)^{2}}\right. \\
& +\left(-8 C_{2}^{3}+8 C_{2} C_{3}+4 C_{3} C_{2}-\frac{32}{9} C_{4}\right) E^{(k)^{3}} \\
& +\left(-\frac{130}{27} C_{5}+16 C_{2}^{4}-16 C_{2}^{3} C_{3}-12 C_{2} C_{3} C_{2}\right.
\end{aligned}
$$


TABLE 4: Comparison of the number of iterations and function evaluations.

\begin{tabular}{|c|c|c|c|c|c|c|c|c|c|c|}
\hline Function & Guess & NM & $\mathrm{CH} 3$ & HM3 & WR3 & M3 & SL4 & $\mathrm{KH} 4$ & M4 I & M4 II \\
\hline \multirow{7}{*}{$f_{1}$} & 5.0 & $6(12)$ & $4(12)$ & $4(12)$ & $4(12)$ & $4(12)$ & $4(12)$ & $3(9)$ & $3(9)$ & $3(9)$ \\
\hline & 4.9 & $6(12)$ & $4(12)$ & $4(12)$ & $4(12)$ & $4(12)$ & $4(12)$ & $3(9)$ & $3(9)$ & $3(9)$ \\
\hline & 5.1 & $6(12)$ & $4(12)$ & $4(12)$ & $4(12)$ & $4(12)$ & $4(12)$ & $3(9)$ & $3(9)$ & $3(9)$ \\
\hline & 4.5 & $6(12)$ & $4(12)$ & $4(12)$ & $4(12)$ & $4(12)$ & $5(15)$ & $4(12)$ & $4(12)$ & $4(12)$ \\
\hline & 5.5 & $6(12)$ & $4(12)$ & $4(12)$ & $4(12)$ & $4(12)$ & $5(15)$ & $4(12)$ & $4(12)$ & $4(12)$ \\
\hline & 4.0 & $7(14)$ & $5(15)$ & $5(15)$ & $5(15)$ & $5(15)$ & $\mathrm{F}$ & $4(12)$ & $4(12)$ & $4(12)$ \\
\hline & 6.0 & $7(14)$ & $5(15)$ & $5(15)$ & $5(15)$ & $5(15)$ & $\mathrm{F}$ & $4(12)$ & $4(12)$ & $4(12)$ \\
\hline \multirow{7}{*}{$f_{2}$} & 2.5 & $8(16)$ & $5(15)$ & $5(15)$ & $5(15)$ & $5(15)$ & $5(15)$ & $5(15)$ & $4(12)$ & $4(12)$ \\
\hline & 2.4 & $8(16)$ & $5(15)$ & $5(15)$ & $5(15)$ & $5(15)$ & $4(12)$ & $4(12)$ & $4(12)$ & $4(12)$ \\
\hline & 2.6 & $8(16)$ & $5(15)$ & $5(15)$ & $5(15)$ & $5(15)$ & $5(15)$ & $5(15)$ & $4(12)$ & $4(12)$ \\
\hline & 1.5 & $8(16)$ & $6(18)$ & $5(15)$ & $5(15)$ & $5(15)$ & $5(15)$ & $6(18)$ & $5(15)$ & $5(15)$ \\
\hline & 3.0 & $9(18)$ & $6(18)$ & $5(15)$ & $6(18)$ & $6(18)$ & $5(15)$ & $5(15)$ & $4(12)$ & $5(15)$ \\
\hline & 1.0 & $10(20)$ & $10(30)$ & $6(18)$ & $7(21)$ & $6(18)$ & $\mathrm{D}$ & $17(51)$ & $9(27)$ & $8(24)$ \\
\hline & 3.5 & $9(18)$ & $6(18)$ & $5(15)$ & $6(18)$ & $6(18)$ & $\mathrm{D}$ & $5(15)$ & $5(15)$ & $5(15)$ \\
\hline \multirow{7}{*}{$f_{3}$} & 0.4 & $6(12)$ & $4(12)$ & $4(12)$ & $4(12)$ & $4(12)$ & $4(12)$ & $4(12)$ & $3(9)$ & $3(9)$ \\
\hline & 0.5 & $7(14)$ & $5(15)$ & $4(12)$ & $4(12)$ & $5(15)$ & $4(12)$ & $4(12)$ & $4(12)$ & $4(12)$ \\
\hline & 0.3 & $7(14)$ & $5(15)$ & $5(15)$ & $4(12)$ & $5(15)$ & $4(12)$ & $4(12)$ & $4(12)$ & $4(12)$ \\
\hline & 1.0 & $8(16)$ & $9(27)$ & $6(18)$ & $5(15)$ & $6(18)$ & $\mathrm{D}$ & $\mathrm{D}$ & $12(36)$ & $6(18)$ \\
\hline & 0.0 & $7(14)$ & $5(15)$ & $5(15)$ & $5(15)$ & $5(15)$ & $5(15)$ & $4(12)$ & $4(12)$ & $4(12)$ \\
\hline & -0.5 & $8(16)$ & $6(18)$ & $6(18)$ & $6(18)$ & $6(18)$ & $\mathrm{D}$ & $5(15)$ & $5(15)$ & $5(15)$ \\
\hline & 0.8 & $8(16)$ & $6(18)$ & $5(15)$ & $5(15)$ & $5(15)$ & $5(15)$ & $5(15)$ & $5(15)$ & $5(15)$ \\
\hline \multirow{7}{*}{$f_{4}$} & 0.3 & $8(16)$ & $6(18)$ & $5(15)$ & $5(15)$ & $5(15)$ & $5(15)$ & $5(15)$ & $5(15)$ & $4(12)$ \\
\hline & 0.1 & $6(12)$ & $4(12)$ & $4(12)$ & $4(12)$ & $4(12)$ & $4(12)$ & $4(12)$ & $4(12)$ & $3(9)$ \\
\hline & 0.2 & $7(14)$ & $5(15)$ & $5(15)$ & $5(15)$ & $5(15)$ & $4(12)$ & $4(12)$ & $4(12)$ & $4(12)$ \\
\hline & 0.6 & $10(20)$ & $7(21)$ & $6(18)$ & $7(21)$ & $6(18)$ & $23(69)$ & F & $9(27)$ & $8(24)$ \\
\hline & -0.4 & $9(18)$ & $6(18)$ & $5(15)$ & $6(18)$ & $6(18)$ & $5(15)$ & $5(15)$ & $5(15)$ & $5(15)$ \\
\hline & 1.3 & $9(18)$ & F & $7(21)$ & $7(21)$ & $14(42)$ & F & $7(21)$ & $6(18)$ & $6(18)$ \\
\hline & -0.9 & $10(20)$ & $7(21)$ & $6(18)$ & $6(18)$ & $6(18)$ & $6(18)$ & $6(18)$ & $5(15)$ & $5(15)$ \\
\hline
\end{tabular}

which gives

$$
\begin{aligned}
& {\left[3 F^{\prime}\left(Y^{(k)}\right)-F^{\prime}\left(X^{(k)}\right)\right]^{-1}} \\
& \quad=\frac{F^{\prime}(\alpha)^{-1}}{2}\left[I+\frac{F_{1}}{2} E^{(k)}+\frac{F_{2}}{2} E^{(k)^{2}}+\frac{F_{3}}{2} E^{(k)^{3}}\right] \\
& \quad+O\left(E^{(k)^{4}}\right),
\end{aligned}
$$

where

$$
\begin{gathered}
F_{1}=4 C_{2}^{2}-2 C_{3}, \\
F_{2}=-8 C_{2}^{3}+8 C_{2} C_{3}+4 C_{3} C_{2}-\frac{32}{9} C_{4},
\end{gathered}
$$

Now from (55) and (50), we have

$$
\begin{aligned}
r= & 2\left[3 F^{\prime}\left(Y^{(k)}\right)-F^{\prime}\left(X^{(k)}\right)\right]^{-1} F^{\prime}\left(Y^{(k)}\right) \\
= & {\left[I+\frac{2}{3} C_{2} E^{(k)}+\left(\frac{4}{3} C_{3}-\frac{2}{3} C_{2}^{2}\right) E^{(k)^{2}}\right.} \\
& \left.+\left(\frac{-4}{3} C_{2} C_{3}+\frac{52}{27} C_{4}\right) E^{(k)^{3}}\right]+O\left(E^{(k)^{4}}\right) .
\end{aligned}
$$

Premultiplying (57) by (48), it can be easily obtained that

$$
\begin{aligned}
r \cdot s= & 2\left[3 F^{\prime}\left(Y^{(k)}\right)-F^{\prime}\left(X^{(k)}\right)\right]^{-1} \\
& \times\left[F^{\prime}\left(Y^{(k)}\right)\right]\left[F^{\prime}\left(X^{(k)}\right)\right]^{-1} F\left(X^{(k)}\right)
\end{aligned}
$$


TABLE 5: Comparison of the norm of the error occurring in the estimates of the exact root with some existing third-order methods.

\begin{tabular}{|c|c|c|c|c|}
\hline Example & Method & $\left\|X_{1}^{(k)}-\alpha\right\|$ & $\left\|X_{2}^{(k)}-\alpha\right\|$ & $\left\|X_{3}^{(k)}-\alpha\right\|$ \\
\hline \multirow{7}{*}{ Example 1} & NR1 & $3.0274 e-5$ & $5.4762 e-16$ & $4.3730 e-48$ \\
\hline & NR2 & $3.0274 e-5$ & $5.4762 e-16$ & $4.3730 e-48$ \\
\hline & NT & $5.9292 e-5$ & $8.2124 e-15$ & $2.9488 e-44$ \\
\hline & $\mathrm{ON}$ & $3.0274 e-5$ & $5.4762 e-16$ & $4.3730 e-48$ \\
\hline & $\mathrm{HZ}$ & $3.0274 e-5$ & $5.4762 e-16$ & $4.3730 e-48$ \\
\hline & NS & $3.0274 e-5$ & $5.4762 e-16$ & $4.3730 e-48$ \\
\hline & M3 I & $2.6220 e-5$ & $2.6977 e-16$ & $4.2171 e-49$ \\
\hline \multirow{7}{*}{ Example 2} & NR1 & $1.7406 e-1$ & $7.1470 e-4$ & $4.5647 e-11$ \\
\hline & NR2 & $1.6363 e-1$ & $5.7582 e-4$ & $2.3870 e-11$ \\
\hline & NT & $2.4632 e-1$ & $3.8429 e-3$ & $1.4191 e-8$ \\
\hline & ON & $1.7034 e-1$ & $6.5958 e-4$ & $3.5877 e-11$ \\
\hline & $\mathrm{HZ}$ & $1.7034 e-1$ & $6.5958 e-4$ & $3.5877 e-11$ \\
\hline & NS & $1.6817 e-1$ & $6.3444 e-4$ & $3.1928 e-11$ \\
\hline & M3 I & $1.0152 e-1$ & $7.0395 e-5$ & $7.8189 e-14$ \\
\hline \multirow{7}{*}{ Example 3} & NR1 & $1.3267 e-3$ & $4.0708 e-10$ & $2.9007 e-30$ \\
\hline & NR2 & $1.3267 e-3$ & $4.0708 e-10$ & $2.9007 e-30$ \\
\hline & NT & $3.0573 e-4$ & $9.6593 e-9$ & $7.2203 e-26$ \\
\hline & $\mathrm{ON}$ & $1.3267 e-3$ & $4.0708 e-10$ & $2.9007 e-30$ \\
\hline & $\mathrm{HZ}$ & $1.3267 e-3$ & $4.0708 e-10$ & $2.9007 e-30$ \\
\hline & NS & $1.3267 e-3$ & $4.0708 e-10$ & $2.9007 e-30$ \\
\hline & M3 I & $1.3282 e-3$ & $4.0708 e-10$ & $2.9007 e-30$ \\
\hline \multirow{7}{*}{ Example 4} & NR1 & $4.9646 e-2$ & $2.0377 e-4$ & $1.9717 e-11$ \\
\hline & NR2 & $4.9260 e-2$ & $1.9653 e-4$ & $1.7731 e-11$ \\
\hline & NT & $6.8356 e-2$ & $9.2740 e-4$ & $3.6811 e-9$ \\
\hline & $\mathrm{ON}$ & $4.9437 e-2$ & $1.9989 e-4$ & $1.8634 e-11$ \\
\hline & $\mathrm{HZ}$ & $4.9453 e-2$ & $1.9989 e-4$ & $1.8634 e-11$ \\
\hline & NS & $4.9460 e-2$ & $2.0022 e-4$ & $1.8727 e-11$ \\
\hline & M3 I & $1.1965 e-1$ & $5.9892 e-4$ & $2.1213 e-10$ \\
\hline \multirow{7}{*}{ Example 5} & NR1 & $4.6620 e-5$ & $8.9529 e-14$ & $6.1663 e-40$ \\
\hline & NR2 & $4.6601 e-5$ & $8.9516 e-14$ & $6.1632 e-40$ \\
\hline & NT & $9.7286 e-5$ & $1.6394 e-12$ & $7.5686 e-36$ \\
\hline & ON & $4.6611 e-5$ & $8.9521 e-14$ & $6.1645 e-40$ \\
\hline & $\mathrm{HZ}$ & $4.6611 e-5$ & $8.9523 e-14$ & $6.1648 e-40$ \\
\hline & NS & $4.6611 e-5$ & $6.9523 e-14$ & $6.1648 e-40$ \\
\hline & M3 I & $2.2192 e-5$ & $1.3119 e-14$ & $1.6090 e-42$ \\
\hline \multirow{7}{*}{ Example 6} & NR1 & $1.4068 e-3$ & $2.3999 e-10$ & $1.3581 e-30$ \\
\hline & NR2 & $1.4068 e-3$ & $2.3999 e-10$ & $1.3581 e-30$ \\
\hline & NT & $3.1325 e-3$ & $5.4369 e-9$ & $3.1678 e-26$ \\
\hline & ON & $1.4068 e-3$ & $2.3999 e-10$ & $1.3581 e-30$ \\
\hline & $\mathrm{HZ}$ & $1.4068 e-3$ & $2.3999 e-10$ & $1.3581 e-30$ \\
\hline & NS & $1.4068 e-3$ & $2.3999 e-10$ & $1.3581 e-30$ \\
\hline & M3 I & $1.4391 e-3$ & $2.6482 e-10$ & $1.8147 e-30$ \\
\hline
\end{tabular}

$$
\begin{aligned}
= & E^{(k)}-\frac{1}{3} C_{2} E^{(k)^{2}}+\left(\frac{2}{3} C_{2}^{2}-\frac{2}{3} C_{3}\right) E^{(k)^{3}} \\
& +\left(-2 C_{2}^{3}-\frac{29}{27} C_{4}+\frac{4}{3} C_{2} C_{3}+\frac{5}{3} C_{3} C_{2}\right) E^{(k)^{4}} \\
& +O\left(E^{(k)^{5}}\right) .
\end{aligned}
$$

Now by virtue of (53) and (58), it can be written as

$$
\begin{aligned}
{\left[\frac{3}{2} I\right.} & \left.-\frac{3}{4} t+\frac{1}{4} t^{2}\right] \cdot r \cdot s \\
& =E^{(k)}+\left(-\frac{43}{27} C_{2}^{3}+C_{3} C_{2}-\frac{1}{9} C_{4}\right) E^{(k)^{4}}+O\left(E^{(k)^{5}}\right) .
\end{aligned}
$$


TABLE 6: Comparison of the norm of the error occurring in the estimates of the exact root with some existing fourth-order methods.

\begin{tabular}{|c|c|c|c|c|}
\hline Example & Method & $\left\|X_{1}^{(k)}-\alpha\right\|$ & $\left\|X_{2}^{(k)}-\alpha\right\|$ & $\left\|X_{3}^{(k)}-\alpha\right\|$ \\
\hline \multirow{4}{*}{ Example 1} & M4 II & $6.0516 e-7$ & $5.2164 e-28$ & $4.1540 e-112$ \\
\hline & BBK & $1.5583 e-6$ & $6.4678 e-26$ & $2.6115 e-103$ \\
\hline & JR & $9.5374 e-7$ & $5.3169 e-27$ & $7.1439 e-108$ \\
\hline & MT & $9.3744 e-7$ & $5.2761 e-27$ & $6.9541 e-108$ \\
\hline \multirow{4}{*}{ Example 2} & M4 II & $1.088 e-1$ & $3.4338 e-6$ & $3.0799 e-24$ \\
\hline & BBK & $9.4638 e-2$ & $9.2598 e-6$ & $8.3045 e-22$ \\
\hline & $J R$ & $9.8464 e-2$ & $5.4080 e-6$ & $4.6299 e-23$ \\
\hline & MT & $2.5630 e-2$ & $1.0710 e-8$ & $8.4788 e-34$ \\
\hline \multirow{4}{*}{ Example 3} & M4 II & $2.0890 e-4$ & $1.1590 e-16$ & $4.0534 e-67$ \\
\hline & BBK & $5.1609 e-4$ & $9.7061 e-15$ & $4.4270 e-59$ \\
\hline & JR & $3.0309 e-4$ & $7.5797 e-16$ & $1.0992 e-63$ \\
\hline & MT & $2.6127 e-4$ & $3.5709 e-16$ & $4.6078 e-65$ \\
\hline \multirow{4}{*}{ Example 4} & M4 II & $1.0527 e-2$ & $4.6896 e-8$ & $1.6605 e-29$ \\
\hline & BBK & $3.5372 e-2$ & $9.9178 e-6$ & $1.0195 e-19$ \\
\hline & JR & $2.4911 e-2$ & $1.5682 e-6$ & $3.3904 e-23$ \\
\hline & MT & $3.0081 e-2$ & $3.5059 e-6$ & $1.1628 e-21$ \\
\hline \multirow{4}{*}{ Example 5} & M4 II & $1.6108 e-6$ & $8.8401 e-24$ & $6.5622 e-93$ \\
\hline & BBK & $5.3003 e-6$ & $2.7113 e-21$ & $1.4597 e-82$ \\
\hline & $J R$ & $2.8482 e-6$ & $1.3655 e-22$ & $5.7544 e-88$ \\
\hline & MT & $3.4874 e-6$ & $3.0141 e-22$ & $1.2962 e-86$ \\
\hline \multirow{4}{*}{ Example 6} & M4 II & $1.7279 e-4$ & $4.5090 e-17$ & $2.0519 e-69$ \\
\hline & BBK & $4.0878 e-4$ & $3.2118 e-15$ & $1.2129 e-59$ \\
\hline & JR & $2.4748 e-4$ & $2.7691 e-16$ & $4.2681 e-64$ \\
\hline & MT & $2.0133 e-4$ & $1.0206 e-16$ & $6.7467 e-66$ \\
\hline
\end{tabular}

Finally using (59), in the second step of method (47) we get

$$
E^{(k+1)}=\left(\frac{43}{27} C_{2}^{3}-C_{3} C_{2}+\frac{1}{9} C_{4}\right) E^{(k)^{4}}+O\left(E^{(k)^{5}}\right)
$$

Thus the proof is completed.

\section{Numerical Testing}

4.1. Single Variable. We consider four test nonlinear functions given in Table 1 to illustrate the accuracy of the new proposed methods. The root of each nonlinear test function is also listed. All the computations reported here have been done using MATMEMATICA 8. Scientific computations in many branches of science and technology demand very high precision degree of numerical precision. We consider the number of decimal places as follows: 200 digits floating point (SetAccuraccy $=200)$ with SetAccuraccy command. The test nonlinear functions are listed in Table 1. Here we compare performances of our new third-order method (15) with the Newton method (NM), Weerakoon and Fernando method (WR3) (12), Homeier method (HM3) (13), and Chun and Kim method (CH3) (14) and our fourth-order methods (M4 I) (24) and (M4 II) (29) with the fourth-order methods (17) (SL4) of [7] and (6) (KH4) of [11]. The results of comparison for the test functions for third- and fourth-order methods are given in Tables 2 and 3, respectively.
In Table 4, numerical tests are given for third-order and fourth-order methods. The test functions have been performed with stopping criterion $\left|f\left(x_{k+1}\right)\right| \leq 10^{-120}$; in addition, the maximum number of iterations is less than 100 . The computational results presented in Table 4 show that the presented method M4 II converges more rapidly than almost all these methods. This new method requires less number of functional evaluations. This means that the new method has better efficiency in computing process as compared to the other methods. For some initial guess, our method gives some bad results as compared to the Newton method but in this case other methods either failed (F)/diverge (D) or gave poor performances as compared to our method M4 II. Also one advantage of our method is that some of the third-order and fourth-order methods failed/diverge for some initial guess but our method does not. So we can conclude that our method M4 II is efficient.

4.2. Multivariate Case. Here we consider six nonlinear system problems. The residual norms for first, second, and third iteration are mentioned in Tables 5 and 6, respectively. We compare our third-order method (31) (M3 I) with the thirdorder methods Algorithm 2.2 (NR1) and Algorithm 2.3 (NR2) of [12], Newton-type method (10) (NT) of [13], NewtonSimpson's method (5) (NS) and Open-Newton's method (6) (ON) of [14], and Algorithm (2) (HZ) of [15] and our fourthorder method (47) (M4 II) with fourth-order method (3.5) 




(a) JM4



(c) SL4

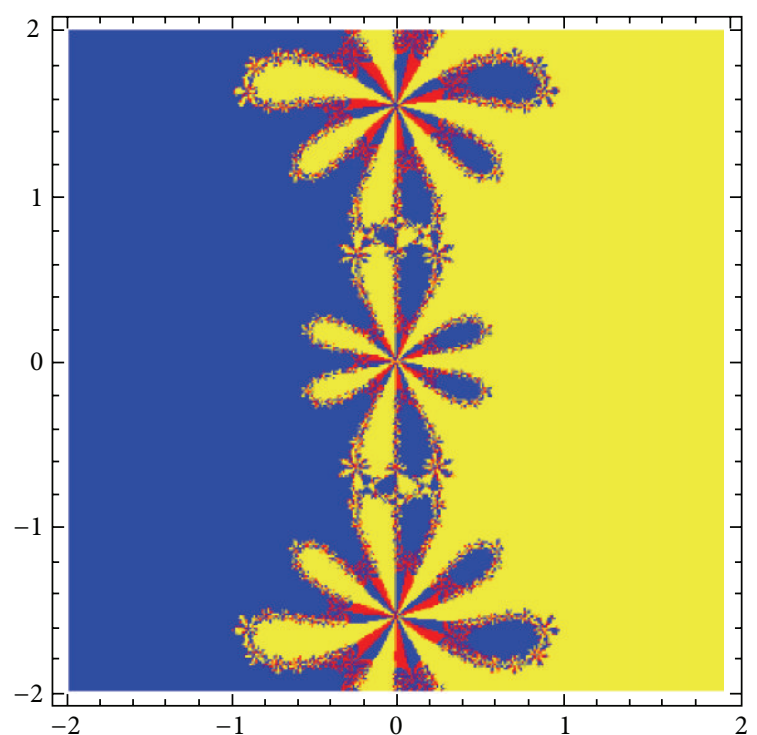

(b) $\mathrm{KH} 4$

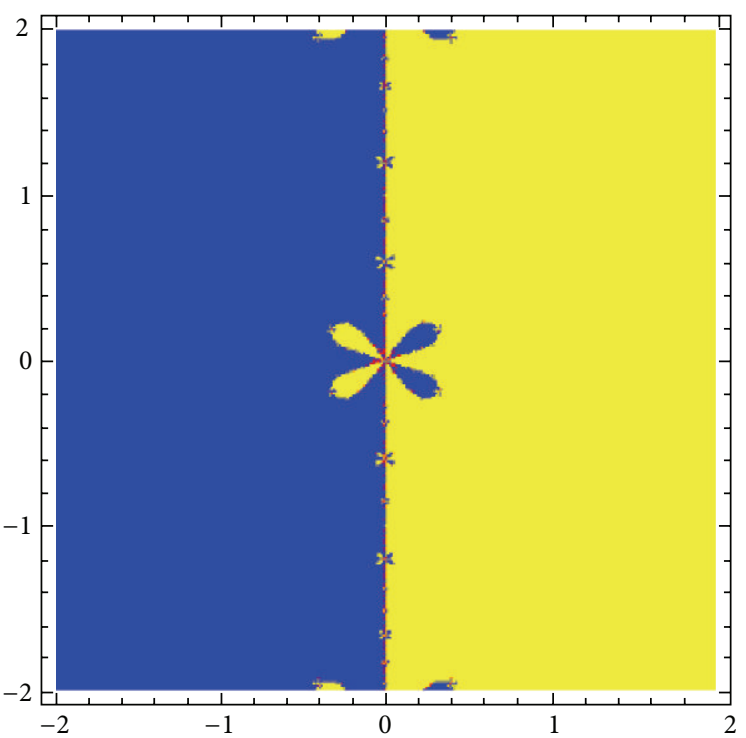

(d) M4 II

Figure 1: Basin of attraction for polynomial $z^{2}-1$.

(BBK) of [16], method (22) (JR) of [17], and method (7) (MT) of [18]. Here the results of comparison for the test functions for third- and fourth-order methods are given in Tables 5 and 6 , respectively.

Consider the following systems of nonlinear equations.

Example 1. We have

$$
\begin{gathered}
x_{1}^{2}-x_{2}-19=0, \\
-x_{1}^{2}+\frac{x_{2}^{3}}{6}+x_{2}-17=0
\end{gathered}
$$

with initial guess $X^{(0)}=(5.1,6.1)^{T}$ and one of its solutions is $\alpha=(5,6)^{T}$. The Jacobian matrix of the above system of equations is given by

$$
\left[\begin{array}{cc}
2 x_{1} & -1 \\
-2 x_{1} & 1+\frac{x_{2}^{2}}{2}
\end{array}\right] .
$$

Example 2. We have

$$
\begin{aligned}
& \log \left(x_{2}\right)-x_{1}^{2}+x_{1} x_{2}=0, \\
& \log \left(x_{1}\right)-x_{2}^{2}+x_{1} x_{2}=0
\end{aligned}
$$






(a) JM4

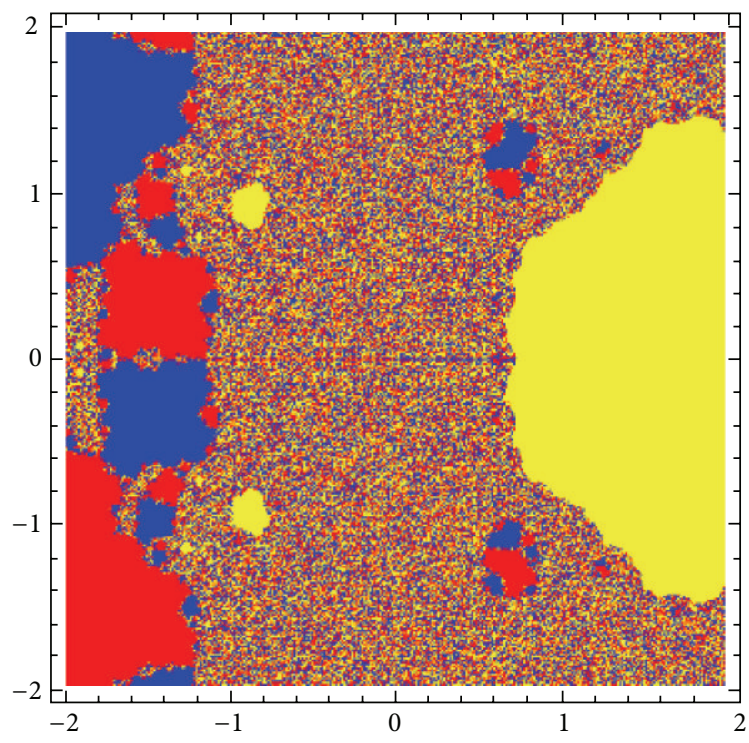

(c) SL4

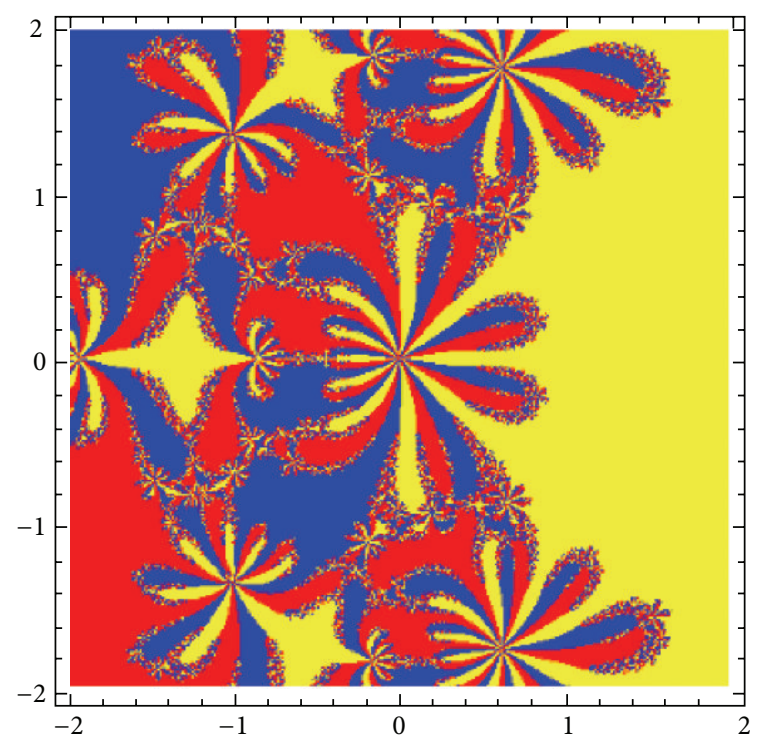

(b) $\mathrm{KH} 4$



(d) M4 II

FIGURE 2: Basin of attraction for polynomial $z^{3}+4 z^{2}-10$.

with initial guess $X^{(0)}=(0.5,1.5)^{T}$ and one of its solutions is $\alpha=(1,1)^{T}$. The Jacobian matrix of the above system of equations is given by

$$
\left[\begin{array}{cc}
-2 x_{1}+x_{2} & x_{1}+\frac{1}{x_{2}} \\
\frac{1}{x_{1}}+x_{2} & x_{1}-2 x_{2}
\end{array}\right] .
$$

Example 3. We have

$$
\begin{aligned}
& x_{2} x_{3}+x_{4}\left(x_{2}+x_{3}\right)=0, \\
& x_{1} x_{3}+x_{4}\left(x_{1}+x_{3}\right)=0, \\
& x_{1} x_{2}+x_{4}\left(x_{1}+x_{2}\right)=0, \\
& x_{1} x_{2}+x_{1} x_{3}+x_{2} x_{3}=1
\end{aligned}
$$






(a) JM4



(c) SL4

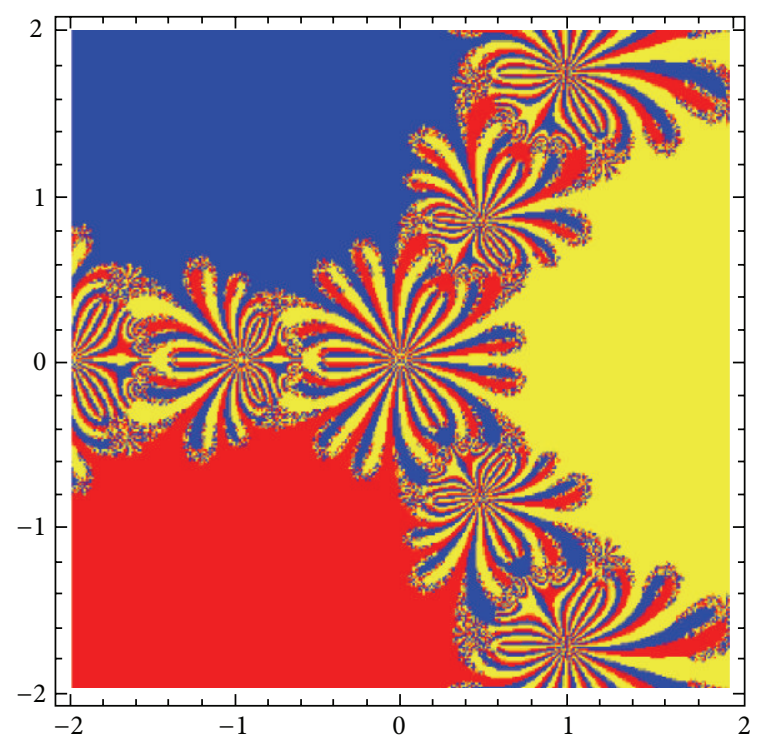

(b) $\mathrm{KH} 4$

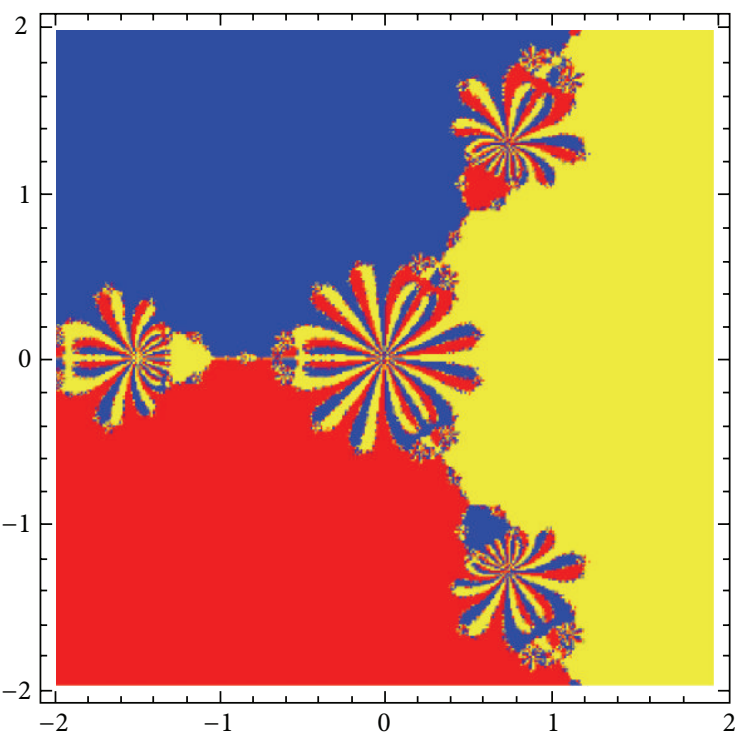

(d) M4 II

FIgURe 3: Basin of attraction for polynomial $z^{3}-1$.

with initial guess $X^{(0)}=(0.5,0.5,0.5,-0.2)^{T}$ and one of its solutions is $\alpha \approx(0.577350,0.577350,0.577350,-0.288675)^{T}$. The Jacobian matrix of the above system of equations is given by

$$
\left[\begin{array}{cccc}
0 & x_{3}+x_{4} & x_{2}+x_{4} & x_{2}+x_{3} \\
x_{3}+x_{4} & 0 & x_{1}+x_{4} & x_{1}+x_{3} \\
x_{2}+x_{4} & x_{1}+x_{4} & 0 & x_{1}+x_{2} \\
x_{2}+x_{3} & x_{1}+x_{3} & x_{1}+x_{2} & 0
\end{array}\right]
$$

Example 4. We have

$$
\begin{gathered}
-\sin \left(x_{1}\right)+\cos \left(x_{2}\right)=0, \\
-\frac{1}{x_{2}}+\left(x_{3}\right)^{x_{1}}=0, \\
e^{x_{1}}-\left(x_{3}\right)^{2}=0
\end{gathered}
$$

with initial guess $X^{(0)}=(1,0.5,1.5)^{T}$ and one of its solutions is $\alpha=(0.9095, \ldots, 0.6612, \ldots, 1.5758, \ldots)^{T}$. The Jacobian 


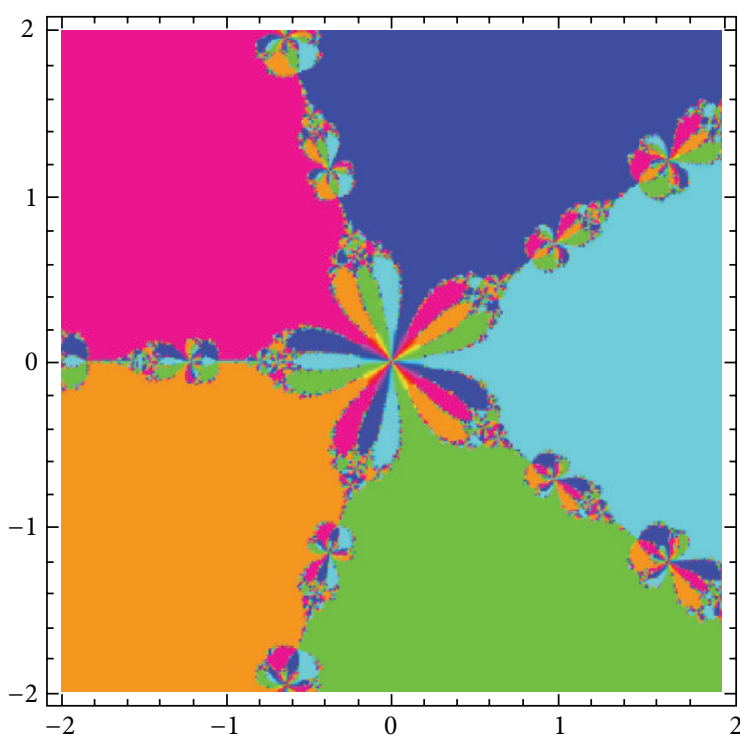

(a) JM4

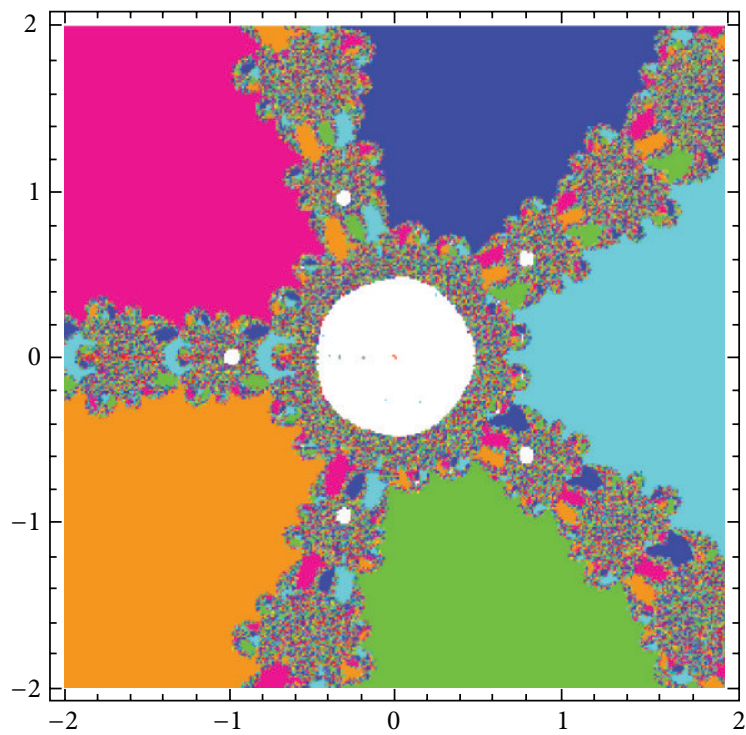

(c) SL4

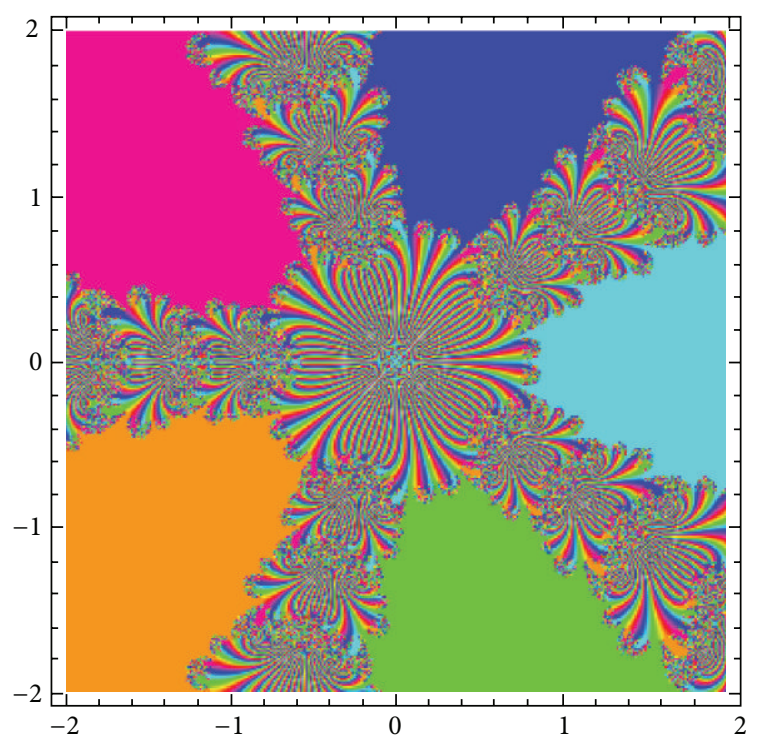

(b) $\mathrm{KH} 4$

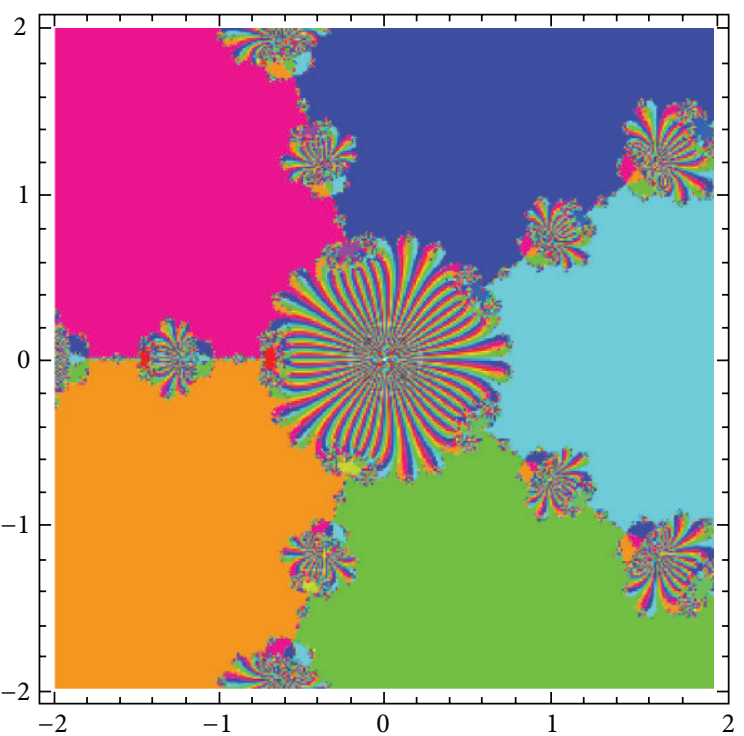

(d) M4 II

FIGURE 4: Basin of attraction for polynomial $z^{5}-1$.

matrix of the above system of equations is given by

$$
\left[\begin{array}{ccc}
-\cos \left(x_{1}\right) & -\sin \left(x_{2}\right) & 0 \\
x_{3}^{x_{1}} \log \left(x_{3}\right) & \frac{1}{x_{2}^{2}} & x_{1} x_{3}^{-1+x_{1}} \\
e^{x_{1}} & 0 & -2 x_{3}
\end{array}\right] .
$$

Example 5. We have

$$
\begin{aligned}
& -e^{-x_{1}}+x_{2}+x_{3}=0, \\
& -e^{-x_{2}}+x_{1}+x_{3}=0, \\
& -e^{-x_{3}}+x_{1}+x_{2}=0
\end{aligned}
$$

with initial guess $X^{(0)}=(-0.8,1.1,1.1)^{T}$ and one of its solutions is $\alpha=(-0.8320, \ldots, 1.1489, \ldots, 1.1489, \ldots)^{T}$. The Jacobian matrix of the above system of equations is given by

$$
\left[\begin{array}{ccc}
e^{-x_{1}} & 1 & 1 \\
1 & e^{-x_{2}} & 1 \\
1 & 1 & e^{-x_{3}}
\end{array}\right]
$$

Example 6. We have

$$
\begin{gathered}
x_{1}^{2}+x_{2}^{2}+x_{3}^{2}-9=0, \\
x_{1} x_{2} x_{3}-1=0, \\
x_{1}+x_{2}-x_{3}^{2}=0
\end{gathered}
$$




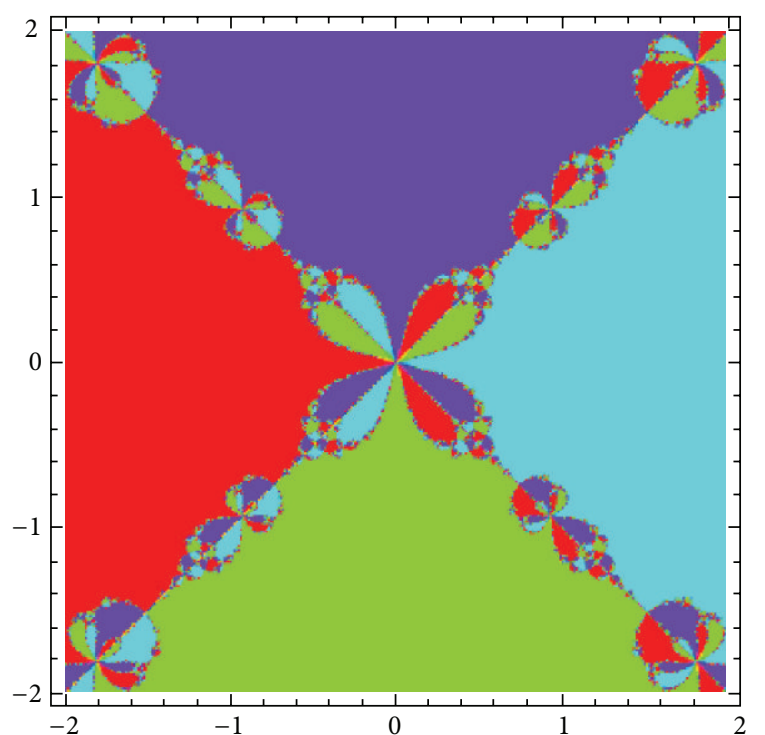

(a) JM4

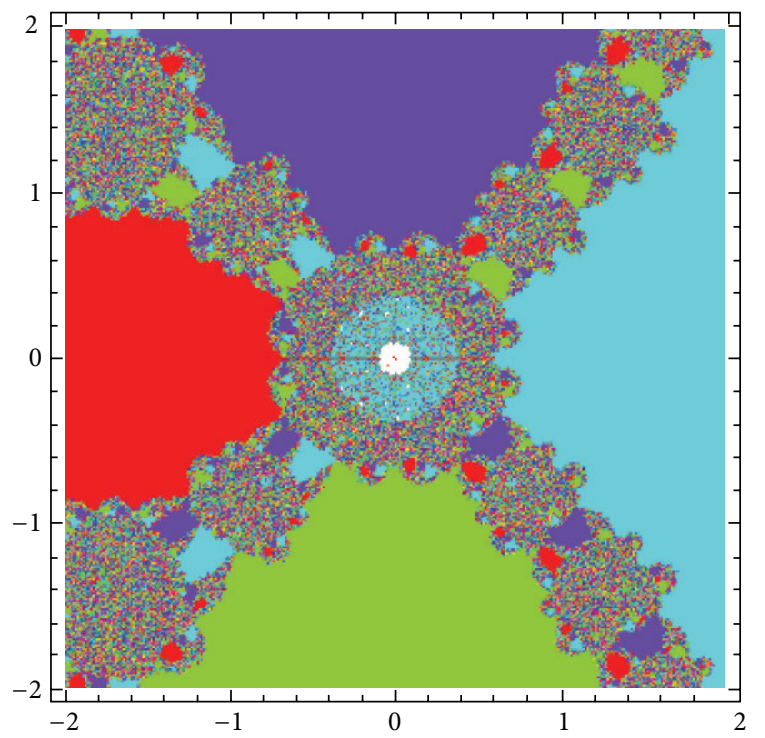

(c) SL4

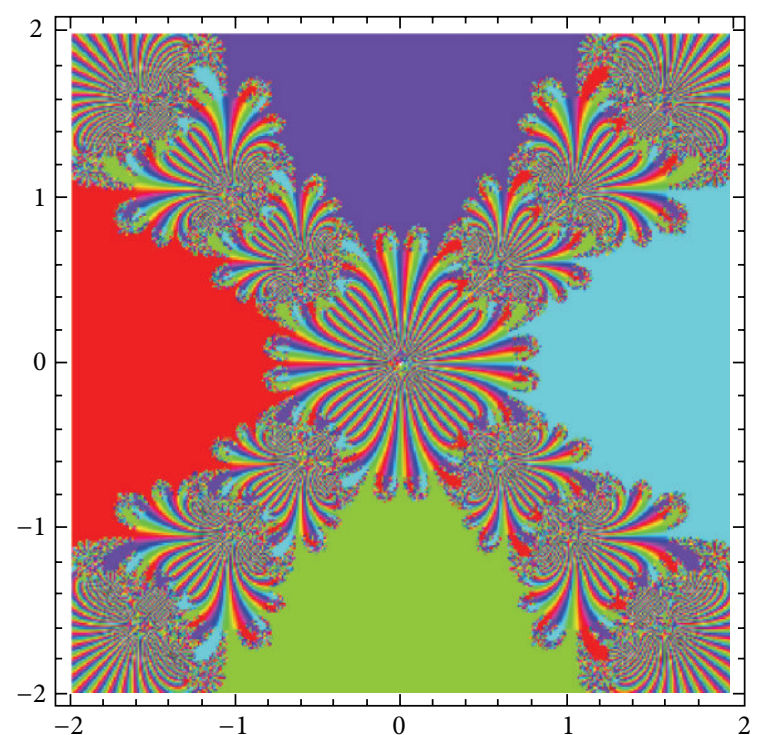

(b) $\mathrm{KH} 4$

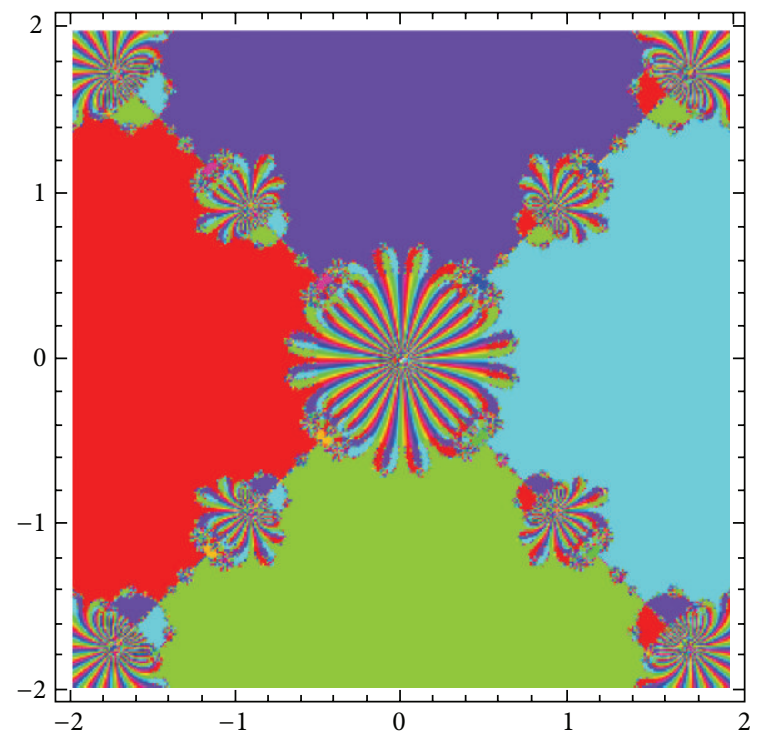

(d) M4 II

FIgURE 5: Basin of attraction for polynomial $z^{4}-1$.

with initial guess $X^{(0)}=(3,1,2)^{T}$ and one of its solutions is $\alpha=(2.2242, \ldots, 0.22838, \ldots, 1.5837, \ldots)^{T}$. The Jacobian matrix of above equations is given by

$$
\left[\begin{array}{ccc}
2 x_{1} & 2 x_{2} & 2 x_{3} \\
x_{2} x_{3} & x_{1} x_{3} & x_{1} x_{2} \\
1 & 1 & -2 x_{3}
\end{array}\right] .
$$

\section{Basin of Attraction}

The basin of attraction for complex Newton's method was first initiated by Cayley [19]. The basin of attraction is a way to view how an algorithm behaves as a function for the various starting points. It is another way to compare the iterative methods. We consider a rectangle $D=[-2,2] \times[-2,2] \in \mathbb{C}$ and assign a colour to each point $z_{0} \in D$ according to the root at which the corresponding iterative method starting from $z_{0}$ converges; for more details one may see $[20,21]$. In this section, the following test functions have been considered for comparison: (i) $z^{2}-1$ (ii) $z^{3}-1$ (iii) $z^{3}+4 z^{2}-10$ (iv) $z^{5}-1$ (v) $z^{4}-1$, and their roots are $\{-1+0 . i, 1+0 . i\},\{1.36523,-2.68262+0.358259 i,-2.68262-$ $0.358259 i\}, \quad\{1,-0.5-0.866025 i,-0.5+0.866025 i\}$, $\{1,-0.809017-0.587785 i, 0.309017+0.951057 i, 0.309017-$ $0.951057 i,-0.809017+0.587785 i\}$ and $\{-1,0-1 . i, 0+1 . i, 1\}$, respectively.

We compare our fourth-order method (30) (M4 II) with Jarratt's method (JM4) of [22], (17) (SL4) of [7], and (6) (KH4) 
of [11]. The results of the comparisons are given in Figures $1,2,3,4$, and 5 . In our first figure, we have performed all the methods to obtain the zeros of the quadratic polynomial $z^{2}-1$. From Figure 1, it is clear that JM4 is best followed by our proposed method M4 II; methods KH4 and SL4 do not perform well. In Figure 2, we have taken a cubic polynomial $z^{3}+4 z^{2}-10$. In this case again JM4 is best with simplest boundary of basin but our proposed method M4 II also shows the simpler boundaries as compared to methods SL4 and KH4. In Figure 3 we have considered a polynomial $z^{3}-1$. It can be seen that, as before, JM4 and M4 II are better than SL4 and KH4. In Figures 4 and 5, we have taken polynomials $z^{5}-1$ and $z^{4}-1$, respectively. In both figures, methods JM4 and M4 II dominate the other two methods, that is, SL4 and KH4. One can note that taking tighter conditions in programming code may develop picture with better quality than these.

From Figures 1-5, it is clear that our proposed fourthorder method and Jarratt's method perform well as compared to the other two fourth-order methods.

\section{Conclusion}

In this paper, we have proposed a class of third- and fourthorder iterative methods for finding simple roots of nonlinear equations. Our third-order method includes methods of Weerakoon and Fernando [1], Homeier [2], and Chun and Kim [3] as particular cases. We have also extended some of our proposed methods from single to multivariate case. A number of numerical examples are given to illustrate the performances of our methods by comparing them with some well existing third- and fourth-order iterative methods. The efficiency of our fourth-order method over some existing fourth-order methods is also supported by basin of attractions.

\section{Conflict of Interests}

The author declares that there is no conflict of interests regarding the publication of this paper.

\section{Acknowledgments}

The author is thankful to the referees and editor for their useful technical comments and valuable suggestions which led to a significant improvement of the paper. The author is also grateful to Dr. F. Soleymani, Iran, and Dr. Sujoy Das, India, for useful discussions.

\section{References}

[1] S. Weerakoon and T. G. I. Fernando, "A variant of Newton's method with accelerated third-order convergence," Applied Mathematics Letters, vol. 13, no. 8, pp. 87-93, 2000.

[2] H. H. H. Homeier, "On Newton-type methods with cubic convergence," Journal of Computational and Applied Mathematics, vol. 176, no. 2, pp. 425-432, 2005.
[3] C. Chun and Y.-I. Kim, "Several new third-order iterative methods for solving nonlinear equations," Acta Applicandae Mathematicae, vol. 109, no. 3, pp. 1053-1063, 2010.

[4] H. T. Kung and J. F. Traub, "Optimal order of one-point and multipoint iteration," Journal of the Association for Computing Machinery, vol. 21, pp. 643-651, 1974.

[5] R. Behl, V. Kanwar, and K. K. Sharma, "Another simple way of deriving several iterative functions to solve nonlinear equations," Journal of Applied Mathematics, vol. 2012, Article ID 294086, 22 pages, 2012.

[6] G. Fernández-Torres and J. Vásquez-Aquino, “Three new optimal fourth-order iterative methods to solve nonlinear equations," Advances in Numerical Analysis, vol. 2013, Article ID 957496, 8 pages, 2013.

[7] F. Soleymani, S. K. Khattri, and S. Karimi Vanani, "Two new classes of optimal Jarratt-type fourth-order methods," Applied Mathematics Letters, vol. 25, no. 5, pp. 847-853, 2012.

[8] W. Gautschi, Numerical Analysis: An Introduction, Birkhäuser, Barton, Mass, USA, 1997.

[9] J. F. Traub, Iterative Methods for the Solution of Equations, Prentice-Hall, Englewood Cliffs, NJ, USA, 1964.

[10] J. P. Jaiswal, "A class of iterative methods for solving nonlinear equation with fourth-order convergence," http://arxiv .org/abs/1307.7334.

[11] S. K. Khattri and S. Abbasbandy, "Optimal fourth order family of iterative methods," Matematichki Vesnik, vol. 63, no. 1, pp. 67$72,2011$.

[12] M. A. Noor and M. Waseem, "Some iterative methods for solving a system of nonlinear equations," Computers \& Mathematics with Applications, vol. 57, no. 1, pp. 101-106, 2009.

[13] M. T. Darvishi and A. Barati, "A third-order Newton-type method to solve systems of nonlinear equations," Applied Mathematics and Computation, vol. 187, no. 2, pp. 630-635, 2007.

[14] A. Cordero and J. R. Torregrosa, "Variants of Newton's method using fifth-order quadrature formulas," Applied Mathematics and Computation, vol. 190, no. 1, pp. 686-698, 2007.

[15] M. Q. Khirallah and M. A. Hafiz, "Novel three order methods for solving a System of nonlinear equations," Bulletin of Society for Mathematical Services and Standards, vol. 1, no. 2, pp. 1-14, 2012.

[16] D. K. R. Babajee, A. Cordero, F. Soleymani, and J. R. Torregrosa, "On a noval fourth-order algorithm for solving systems of nonlinear equations," Journal of Applied Mathematics, vol. 2012, Article ID 165452, 12 pages, 2012.

[17] J. R. Sharma, R. K. Guha, and R. Sharma, "An efficient fourth order weighted-Newton method for systems of nonlinear equations," Numerical Algorithms, vol. 62, no. 2, pp. 307-323, 2013.

[18] M. T. Darvishi and A. Barati, "A fourth-order method from quadrature formulae to solve systems of nonlinear equations," Applied Mathematics and Computation, vol. 188, no. 1, pp. 257261, 2007.

[19] A. Cayley, "The-Newton-Fourier imaginary problem," American Journal of Mathematics, vol. 2, Article 97, 1879.

[20] M. Scott, B. Neta, and C. Chun, "Basin attractors for various methods," Applied Mathematics and Computation, vol. 218, no. 6, pp. 2584-2599, 2011. 
[21] B. Neta, M. Scott, and C. Chun, "Basins of attraction for several methods to find simple roots of nonlinear equations," Applied Mathematics and Computation, vol. 218, no. 21, pp. 10548-10556, 2012.

[22] P. Jarratt, "Some efficient fourth order multipoint methods for solving equations," BIT Numerical Mathematics, vol. 9, pp. 119$124,1969$. 


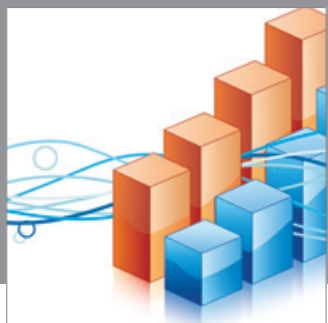

Advances in

Operations Research

mansans

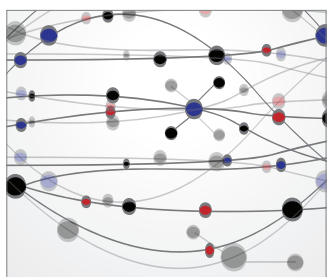

The Scientific World Journal

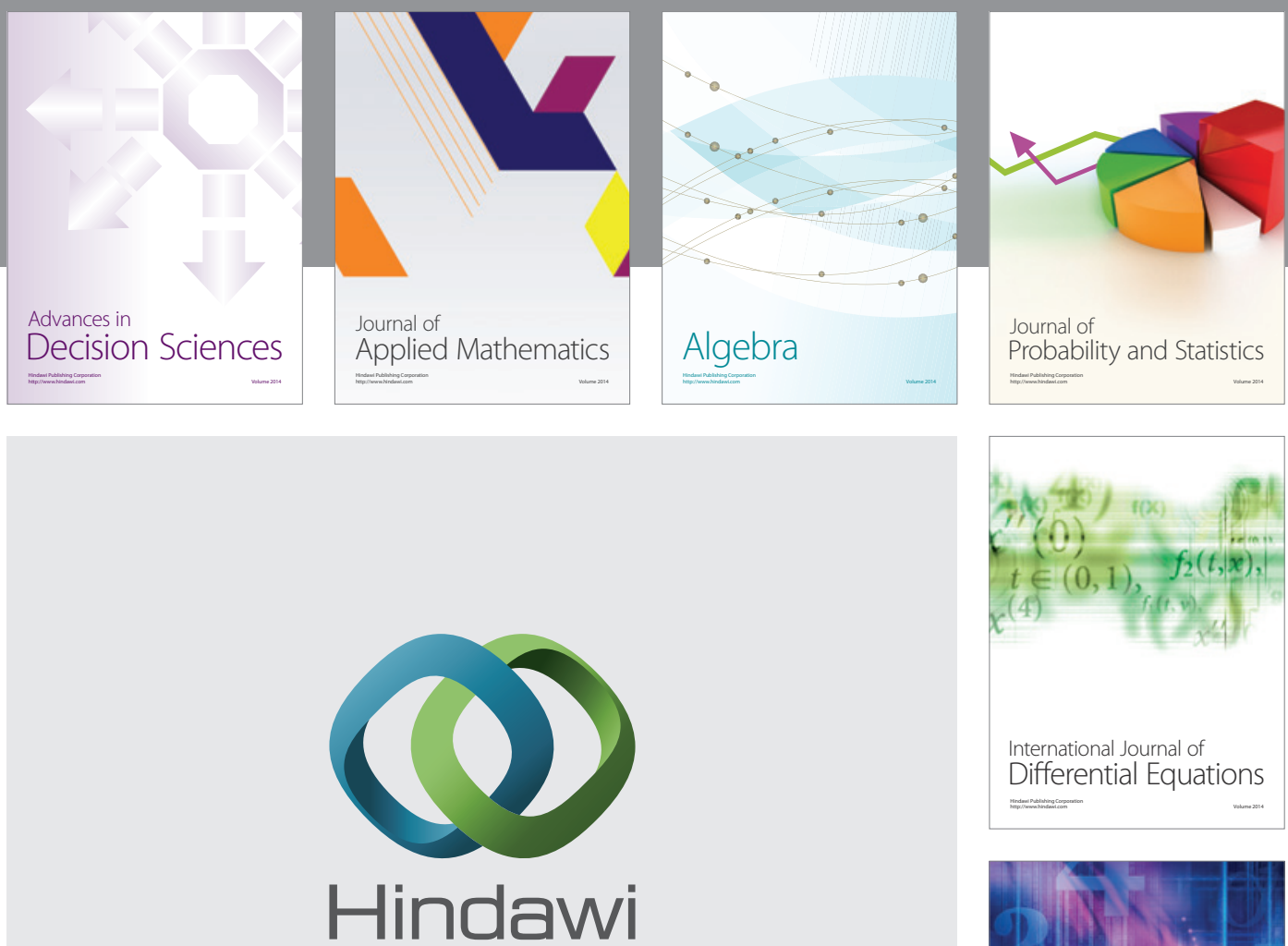

Submit your manuscripts at http://www.hindawi.com
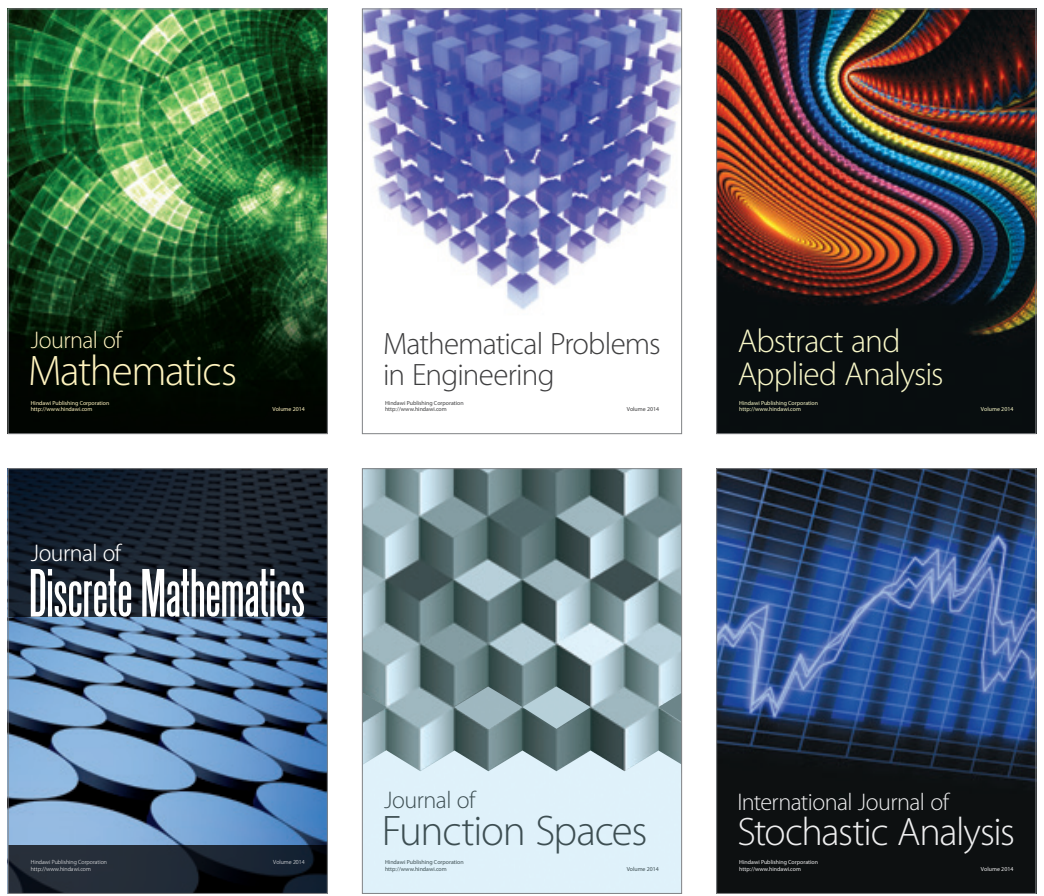

Journal of

Function Spaces

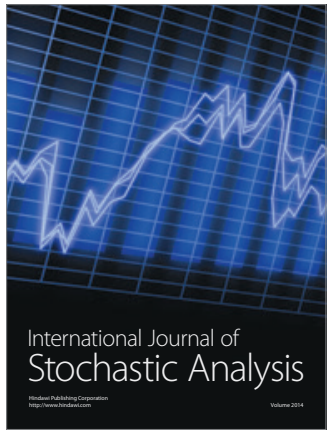


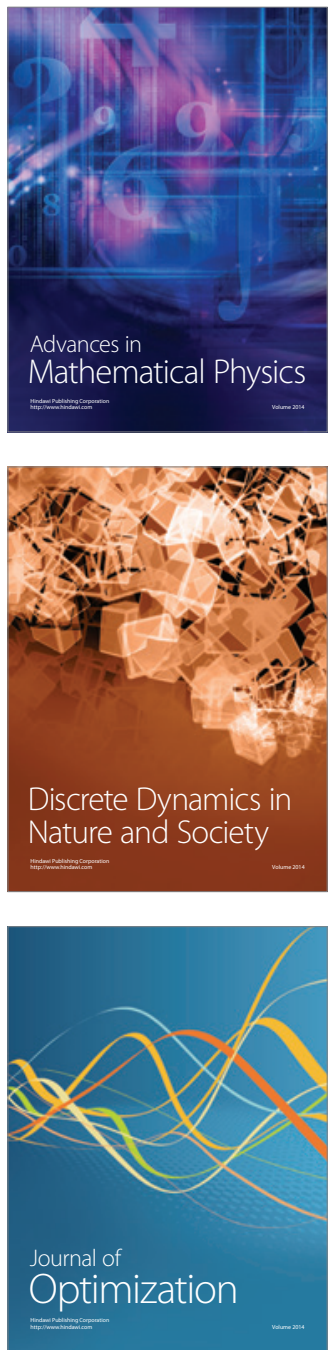